\title{
On the Number of Matchings in Regular Graphs
}

\author{
S. Friedland, E. Krop ${ }^{\dagger}$ and K. Markström ${ }^{\ddagger}$ \\ Submitted: Jan 18, 2008; Accepted: Aug 22, 2008; Published: Aug 31, 2008 \\ Mathematics Subject Classification: 05A15, 05A16, 05C70, 05C80, 05C88, 82B20
}

\begin{abstract}
For the set of graphs with a given degree sequence, consisting of any number of $2^{\prime} s$ and $1^{\prime} s$, and its subset of bipartite graphs, we characterize the optimal graphs who maximize and minimize the number of $m$-matchings.

We find the expected value of the number of $m$-matchings of $r$-regular bipartite graphs on $2 n$ vertices with respect to the two standard measures. We state and discuss the conjectured upper and lower bounds for $m$-matchings in $r$-regular bipartite graphs on $2 n$ vertices, and their asymptotic versions for infinite $r$-regular bipartite graphs. We prove these conjectures for 2-regular bipartite graphs and for $m$-matchings with $m \leq 4$.
\end{abstract}

Keywords and phrases: Partial matching and asymptotic growth of average matchings for $r$-regular bipartite graphs, asymptotic matching conjectures.

\section{Introduction}

Let $G=(V, E)$ be an undirected graph with the set of vertices $V$ and the set of edges $E$. An $m$-matching $M \subset E$, is a set of $m$ distinct edges in $E$, such that no two edges have a common vertex. We say that $M$ covers $U \subseteq V$, $\# U=2 \# M$, if the set of vertices incident to $M$ is $U$. Denote by $\phi(m, G)$ the number of $m$-matchings in $G$. If $\# V$ is even then $\frac{\# V}{2}$-matching is called a perfect matching, or 1-factor of $G$, and $\phi\left(\frac{\# V}{2}, G\right)$ is the number of 1-factors in $G$. For an infinite graph $G=(V, E)$, a match $M \subset E$ is a match of density

*Department of Mathematics, Statistics and Computer Science, University of Illinois at Chicago, Chicago, Illinois 60607-7045, USA (friedlan@uic.edu).

${ }^{\dagger}$ Department of Mathematics, Statistics and Computer Science, University of Illinois at Chicago, Chicago, Illinois 60607-7045, USA (ekrop1@math.uic.edu).

${ }^{\ddagger}$ Department of Mathematics and Mathematical Statistics, UmeåUniversity, SE-901 87 Umeå, Sweden 
$p \in[0,1]$, if the proportion of vertices in $V$ covered by $M$ is $p$. Then the $p$-matching entropy of $G$ is defined as

$$
h_{G}(p)=\limsup _{k \rightarrow \infty} \frac{\log \phi\left(m_{k}, G_{k}\right)}{\# V_{k}},
$$

where $G_{k}=\left(E_{k}, V_{k}\right), k \in \mathbb{N}$ is a sequence of finite graphs converging to $G$, and $\lim _{k \rightarrow \infty} \frac{2 m_{k}}{\# V_{k}}=p$. See [4] for details.

The object of this paper is twofold. First we consider the family $\Omega(n, k)$, the set of simple graphs on $n$ vertices with $2 k$ vertices of degree 1 and $n-2 k$ vertices of degree 2. Let $\Omega_{\mathrm{bi}}(n, k) \subset \Omega(n, k)$ be the subset of bipartite graphs. For each $m \in[2, n] \cap \mathbb{N}$ we characterize the optimal graphs which maximize and minimize $\phi(m, G), m \geq 2$ for $G \in \Omega(n, k)$ and $G \in \Omega_{\mathrm{bi}}(n, k)$. It turns out the optimal graphs do not depend on $m$ but on $n$ and $k$. Furthermore, the graphs with the maximal number of $m$-matchings, are bipartite.

Second, we consider $\mathcal{G}(2 n, r)$, the set of simple bipartite $r$-regular graphs on $2 n$ vertices, where $n \geq r$. Denote by $C_{l}$ a cycle of length $l$ and by $K_{r, r}$ the complete bipartite graph with $r$-vertices in each group. For a nonnegative integer $q$ and a graph $G$ denote by $q G$ the disjoint union of $q$ copies of $G$. Let

$$
\begin{aligned}
& \lambda(m, n, r):=\min _{G \in \mathcal{G}(2 n, r)} \phi(m, G), \quad \Lambda(m, n, r):=\max _{G \in \mathcal{G}(2 n, r)} \phi(m, G), \\
& m=1, \ldots, n .
\end{aligned}
$$

Our results on 2-regular graphs yield.

$$
\begin{aligned}
& \lambda(m, n, 2)=\phi\left(m, C_{2 n}\right), \\
& \Lambda(m, 2 q, 2)=\phi\left(m, q K_{2,2}\right), \quad \Lambda(m, 2 q+3,2)=\phi\left(m, q K_{2,2} \cup C_{6}\right), \\
& \text { for } m=1, \ldots, n .
\end{aligned}
$$

The equality $\Lambda(m, 2 q, 2)=\phi\left(m, q K_{2,2}\right)$ inspired us to conjecture the Upper Matching Conjecture, abbreviated here as UMC:

$$
\Lambda(m, q r, r)=\phi\left(m, q K_{r, r}\right) \text { for } m=1, \ldots, q r
$$

For the value $m=q r$ the UMC follows from Bregman's inequality [1]. For the value $r=3$ the UMC holds up to $q \leq 8$. The results of [4] support the validity of the above conjecture for $r=3,4$ and large values of $n=q r$. As in the case $r=2$ we conjecture that for any nonbipartite $r$-regular graph on $2 n$ vertices $\phi(m, G) \leq \Lambda(m, n, r)$ for $m=1, \ldots, n$.

It is useful to consider $\mathcal{G}_{\text {mult }}(2 n, r) \supset \mathcal{G}(2 n, r)$, the set of $r$-regular bipartite graphs on $2 n$ vertices, where multiple edges are allowed. Observe that $\mathcal{G}_{\text {mult }}(2, r)=\left\{H_{r}\right\}$, where $H_{r}$ is the $r$-regular bipartite multigraph on 2 vertices. Let

$$
\begin{aligned}
\mu(m, n, r):=\min _{G \in \mathcal{G}_{\text {mult }}(2 n, r)} \phi(m, G), \quad M(m, n, r) & :=\max _{G \in \mathcal{G}_{\text {mult }}(2 n, r)} \phi(m, G), \\
m & =1, \ldots, n, 2 \leq r \in \mathbb{N} .
\end{aligned}
$$


It is straightforward to show that

$$
M(m, n, r)=\phi\left(m, n H_{r}\right)=\left(\begin{array}{c}
n \\
m
\end{array}\right) r^{m}, \quad m=1, \ldots, n .
$$

Hence for most of the values of $m, \Lambda(m, n, r)<M(m, n, r)$. On the other hand, as in the case of $\Omega(n, k)$, it is plausible to conjecture that $\lambda(m, n, r)=\mu(n, m, r)$ for all allowable values $m, n$ and $r \geq 3$.

It was shown by Schrijver [10] that for $r \geq 3$

$$
\phi(n, G) \geq\left(\frac{(r-1)^{r-1}}{r^{r-2}}\right)^{n}, \quad \text { for all } G \in \mathcal{G}_{\text {mult }}(2 n, r) .
$$

This lower bound is asymptotically sharp, and in [11] Wanless proved that the bound is sharp when restricted to 0/1-matrices as well. In the first version of this paper we stated the conjectured lower bound

$$
\phi(m, G) \geq\left(\begin{array}{c}
n \\
m
\end{array}\right)^{2}\left(\frac{n r-m}{n r}\right)^{r n-m}\left(\frac{m r}{n}\right)^{m}
$$

for all $G \in \mathcal{G}_{\text {mult }}(2 n, r)$ and $m=1, \ldots, n$.

Note that for $m=n$ the above inequality reduces to (1.7). Our computations suggest a slightly stronger version of the above conjecture (7.1).

Recently Gurvits [6] improved (1.7) to

$$
\phi(n, G) \geq \frac{r !}{r^{r}}\left(\frac{r}{r-1}\right)^{r(r-1)}\left(\frac{(r-1)^{r-1}}{r^{r-2}}\right)^{n}, \quad G \in \mathcal{G}_{\text {mult }}(2 n, r) .
$$

In [3] the authors were able to generalize the above inequality to partial matching, which are very close to optimal results asymptotically, see [4] and below.

The next question we address is the expected value of the number of $m$-matchings in $\mathcal{G}_{\text {mult }}(2 n, r)$. There are two natural measures $\mu_{1, n, r}, \mu_{2, n, r}$ on $\mathcal{G}_{\text {mult }}(2 n, r),[7$, Ch.9] and [8, Ch.8]. Let $E_{i}(m, n, r)$ be the expected value of $\phi(m, G)$ with respect to the measure $\mu_{i, n, r}$ for $i=1,2$. In this paper we show that

$$
\begin{gathered}
\lim _{k \rightarrow \infty} \frac{\log E_{i}\left(m_{k}, n_{k}, r\right)}{2 n_{k}}=g h_{r}(p), \text { for } i=1,2, \\
\text { if } \lim _{k \rightarrow \infty} n_{k}=\lim _{k \rightarrow \infty} m_{k}=\infty, \quad \text { and } \lim _{k \rightarrow \infty} \frac{m_{k}}{n_{k}}=p \in[0,1], \\
g h_{r}(p):=\frac{1}{2}\left(p \log r-p \log p-2(1-p) \log (1-p)+(r-p) \log \left(1-\frac{p}{r}\right)\right) .
\end{gathered}
$$

In view of (1.10) the inequalities (1.7) and (1.9) give the best possible exponential term in the asymptotic growth with respect to $n$, as stated in [10]. Similarly, the conjectured inequality (1.8), if true, gives the best possible exponential term in the asymptotic growth with respect to $n$, and $p=\frac{m}{n}$. 
For $p \in[0,1]$ let $\operatorname{low}_{r}(p)$ be the infimum of $\liminf _{k \rightarrow \infty} \frac{\log \mu\left(m_{k}, n_{k}, r\right)}{2 n_{k}}$ over all sequences satisfying (1.11). Hence $h_{G}(p) \geq \operatorname{low}_{r}(p)$ for any infinite bipartite $r$-regular graph. Clearly $\operatorname{low}_{r}(p) \leq g h_{r}(p)$. We conjecture

$$
\operatorname{low}_{r}(p)=g h_{r}(p)
$$

(1.2) implies the validity of this conjecture for $r=2$. The results of [3] imply the validity of this conjecture for each $p=\frac{r}{r+s}, s=0,1, \ldots$ and any $r \geq 3$. In [4] we give lower bounds on $\operatorname{low}_{r}(p)$ for each $p \in[0,1]$ and $r \geq 3$ which are very close to $g h_{r}(p)$.

We stated first our conjectures in the first version of this paper in Spring 2005. Since then the conjectured were restated in $[3,4]$ and some progress was made toward validations of these conjectures.

We now survey briefly the contents of this paper. In $\S 2$ we give sharp bounds for the number of $m$-matchings for general and bipartite 2-regular graphs. In $\S 3$ we generalize these results to $\Omega(n, k)$. In $\S 4$ we find the average of $m$-matchings in $r$-regular bipartite graphs with respect to the two standard measures. We also show the equality (1.10). In $\S 5$ we discuss the Asymptotic Lower Matching Conjecture. In $\S 6$ we discuss briefly upper bounds for matchings in $r$-regular bipartite graphs. In $\S 7$ we bring computational results for regular bipartite graphs on at most 36 vertices. We verified for many of these graphs the LMC and UMC. Among the cubic bipartite graphs on at most 24 vertices we characterized the graphs with the maximal number of $m$-matching in the case $n$ is not divisible by 3 . In $\S 8$ we find closed formulas for $\phi(m, G)$ for $m=2,3,4$ and any $G \in \mathcal{G}(2 n, r)$. It turns out that $\phi(2, G)$ and $\phi(3, G)$ depend only on $n$ and $r . \phi(4, G)=p_{1}(n, r)+a_{4}(G)$, where

$a_{4}(G)$ is the number of 4 cycles in $G . a_{4}(G) \leq \frac{n r(r-1)^{2}}{4}$ and equality holds if and only if $G=q K_{r, r}$.

\section{Sharp bounds for 2-regular graphs}

In this section we find the maximal and the minimal numbers of $m$-matchings of 2-regular bipartite and non-bipartite graphs on $n$ vertices. For the bipartite case this problem was studied, and in fact solved, in [12]. First we introduce the following partial order on the algebra of polynomials with real coefficients, denoted by $\mathbb{R}[x]$. By $0 \in \mathbb{R}[x]$ we denote the zero polynomial.

For any two polynomials $f(x), g(x) \in \mathbb{R}[x]$ we let $g(x) \succeq f(x)$, or $g \succeq f$, if and only if all the coefficients of $g(x)-f(x)$ are nonnegative. We let $g \succ f$ if $g \succeq f$ and $g \neq f$. Let $\mathbb{R}_{+}[x]$ be the cone of all polynomial with nonnegative coefficients in $\mathbb{R}[x]$. Then $\mathbb{R}_{+}[x]+\mathbb{R}_{+}[x]=\mathbb{R}_{+}[x] \mathbb{R}_{+}[x]=\mathbb{R}_{+}[x]$. Furthermore, if $g_{1} \succeq f_{1} \succ 0, g_{2} \succeq f_{2} \succ 0$ then $g_{1} g_{2} \succ f_{1} f_{2}$ unless $g_{1}=f_{1}$ and $g_{2}=f_{2}$.

Denote $\langle n\rangle:=\{1, \ldots, n\}$. Let $G=(V, E)$ be a graph on $n$ vertices. We will identify $V$ with $\langle n\rangle$. We agree that $\phi(0, G)=1$. Denote by $\Phi_{G}(x)$ the generating matching polynomial

$$
\Phi_{G}(x):=\sum_{m=0}^{\left\lfloor\frac{n}{2}\right\rfloor} \phi(m, G) x^{m}=\sum_{m=0}^{\infty} \phi(m, G) x^{m} .
$$


It is straightforward to show that for any two graphs $G=(V, E), G^{\prime}=\left(V^{\prime}, E^{\prime}\right)$ we have the equality

$$
\Phi_{G \cup G^{\prime}}(x)=\Phi_{G}(x) \Phi_{G^{\prime}}(x) .
$$

Denote by $P_{k}$ a path on $k$ vertices: $1-2-3-\cdots-k$. View each match as an edge. Then an $m$-matching of $P_{k}$ is composed of $m$ edges and $k-2 m$ vertices. Altogether $k-m$ objects. Hence the number of $m$-matchings is equal to the number of different ways to arrange $m$ edges and $k-2 m$ vertices on a line. Thus

$$
\begin{aligned}
& \phi\left(P_{k}, m\right)=\left(\begin{array}{c}
k-m \\
m
\end{array}\right) \text { for } m=1, \ldots,\left\lfloor\frac{k}{2}\right\rfloor, \\
& p_{k}(x):=\Phi_{P_{k}}(x)=\sum_{m=0}^{\left\lfloor\frac{k}{2}\right\rfloor}\left(\begin{array}{c}
k-m \\
m
\end{array}\right) x^{m}=\sum_{m=0}^{\infty}\left(\begin{array}{c}
k-m \\
m
\end{array}\right) x^{m} .
\end{aligned}
$$

It is straightforward to see that $p_{k}(x)$ satisfy the recursive relation

$$
\begin{aligned}
& p_{k}(x)=p_{k-1}(x)+x p_{k-2}(x), \quad k=2, \ldots, \\
& \text { where } p_{1}(x)=1, \Phi_{P_{0}}(x):=p_{0}(x)=1 .
\end{aligned}
$$

Indeed, $p_{2}(x)=1+x=p_{1}(x)+x p_{0}(x)$. Assume that $k \geq 3$. All matchings of $P_{k}$, where the vertex $k$ is not in the matching, generate the polynomial $p_{k-1}(x)$. All matchings of $P_{k}$, where the vertex $k$ is in the matching, generate the polynomial $x p_{k-2}(x)$. Hence the above equality holds. Observe next

$$
q_{k}(x):=\Phi_{C_{k}}(x)=p_{k}(x)+x p_{k-2}(x), k=3, \ldots
$$

Indeed, $p_{k}(x)$ is the contribution from all matching which does not include the matching $1-k$. The polynomial $x p_{k-2}(x)$ corresponds to all matchings which include the matching $1-k$.

Use (2.5) to deduce

$$
\begin{aligned}
& q_{k}(x)=q_{k-1}(x)+x q_{k-2}(x), \quad k=3, \ldots, \\
& \text { where } \Phi_{C_{2}}:=q_{2}(x)=1+2 x, \Phi_{C_{1}}:=q_{1}(x)=1 .
\end{aligned}
$$

Note that we identify $C_{2}$ with the 2-regular bipartite multigraph $H_{2}$. It is useful to consider (2.5) for $k=1,0$ and (2.6) for $k=2$. This yields the equalities:

$$
\Phi_{P_{-1}}(x)=p_{-1}=0, \Phi_{P_{-2}}(x)=p_{-2}=\frac{1}{x}, \Phi_{C_{0}}(x)=q_{0}=2 .
$$

Clearly

$$
\begin{array}{r}
p_{-1}=0 \prec p_{0}=p_{1}=q_{1}=1 \prec q_{0}=2, p_{2}=1+x \prec q_{2}=p_{3}=1+2 x, \\
p_{n} \prec q_{n} \prec p_{n+1} \text { for all integers } n \geq 3 .
\end{array}
$$


Theorem 2.1 Let $i \leq j$ be nonnegative integers. Then

$$
\Phi_{C_{i}}(x) \Phi_{C_{j}}(x)-\Phi_{C_{i+j}}(x)=(-1)^{i} x^{i} \Phi_{C_{j-i}}(x) .
$$

In particular, $\Phi_{C_{i}}(x) \Phi_{C_{j}}(x) \succ \Phi_{C_{i+j}}(x)$ if $i$ is even, and $\Phi_{C_{i}}(x) \Phi_{C_{j}}(x) \prec \Phi_{C_{i+j}}(x)$ if $i$ is odd.

Proof. We use the notation $q_{k}=\Phi_{C_{k}}$ for $k \geq 0$. The case $i=0$ follows immediately from $q_{0}=2$. The case $i=1$ follows from $q_{1}=1$ and the identity (2.7) for $k \geq 2$ : $1 q_{j}-q_{j+1}=q_{j}-\left(q_{j}+x q_{j-1}\right)=-x q_{j-1}$. We prove the other cases of the theorem by induction on $i$. Assume that the theorem holds for $i \leq l$, where $l \geq 1$. Let $i=l+1$. Then for $j \geq l+1$ use (2.7) for $k \geq 2$ and the induction hypothesis for $i=l$ and $i=l-1$ to obtain:

$$
\begin{aligned}
q_{l+1} q_{j}-q_{l+1+j} & =\left(q_{l}+x q_{l-1}\right) q_{j}-\left(q_{l+j}+x q_{l-1+j}\right)=q_{l} q_{j}-q_{l+j}+x\left(q_{l-1} q_{j}-q_{l-1+j}\right) \\
& =(-1)^{l+1} x^{l}\left(-q_{j-l}+q_{j-l+1}\right)=(-1)^{l+1} x^{l+1} q_{j-l-1} .
\end{aligned}
$$

Hence (2.11) holds. Since $q_{k} \succ 0$ for $k \geq 0$ (2.11) implies the second part of the theorem.

Theorem 2.2 Let $G$ be a 2-regular graph on $n \geq 4$ vertices. Then

$$
\begin{aligned}
& \Phi_{G}(x) \preceq \Phi_{C_{4}}(x)^{\frac{n}{4}} \text { if } 4 \mid n \\
& \Phi_{G}(x) \preceq \Phi_{C_{4}}(x)^{\frac{n-5}{4}} \Phi_{C_{5}}(x) \text { if } 4 \mid n-1, \\
& \Phi_{G}(x) \preceq \Phi_{C_{4}}(x)^{\frac{n-6}{4}} \Phi_{C_{6}}(x) \text { if } 4 \mid n-2, \\
& \Phi_{G}(x) \preceq \Phi_{C_{4}}(x)^{\frac{n-7}{4}} \Phi_{C_{7}}(x) \text { if } 4 \mid n-3, \\
& \Phi_{G}(x) \succeq \Phi_{C_{3}}(x)^{\frac{n}{3}} \text { if } 3 \mid n \\
& \Phi_{G}(x) \succeq \Phi_{C_{3}}(x)^{\frac{n-4}{3}} \Phi_{C_{4}}(x) \text { if } 3 \mid n-1, \\
& \Phi_{G}(x) \succeq \Phi_{C_{3}}(x)^{\frac{n-5}{3}} \Phi_{C_{5}}(x) \text { if } 3 \mid n-2 .
\end{aligned}
$$

Equalities in (2.12-2.15) hold if and only if $G$ is either a union of copies of $C_{4}$, or a union of copies of $C_{4}$ and a copy of $C_{i}$ for $i=5,6,7$, respectively. Equalities in (2.16-2.18) hold if and only if $G$ is either a union of copies of $C_{3}$, or a union of copies of $C_{3}$ and a copy of $C_{i}$ for $i=4,5$, respectively.

Assume that $n$ is even and $G$ is a bipartite 2-regular multigraph. Then $\Phi_{G}(x) \succeq$ $\Phi_{C_{n}}(x)$. Equality holds if and only if $G=C_{n}$.

Proof. Recall that any 2-regular graph $G$ is a union of cycles of order 3 at least. Use (2.2) to deduce that the matching polynomial of $G$ is the product of the matching polynomials of the corresponding cycles.

We discuss first the upper bounds on $\Phi_{G}$. If $C_{i}$ and $C_{j}$ are two odd cycles Theorem 2.1 yields that $q_{i} q_{j} \prec q_{i+j}$, where $C_{i+j}$ is an even cycle. To find the upper bound on $\Phi_{G}$ 
we may assume that $G$ contains at most one odd cycle. For all cycles $C_{l}$, where $l \geq 8$ Theorem 2.1 yields the inequality $q_{l} \prec q_{4} q_{l-4}$. Use repeatedly this inequality, until we replaced the products of different $q_{l}$ with products involving $q_{4}, q_{6}$ and perhaps one factor of the form $q_{i}$ where $i \in\{3,5,7\}$. Use (2.11) to obtain the inequality:

$$
q_{4}^{3}=q_{4}\left(q_{8}+2 x^{4}\right)=q_{12}+3 x^{4} q_{4} \succ q_{12}+2 x^{6}=q_{6}^{2} .
$$

Hence we may assume that $G$ contains at most one cycle of length 6 . If $n$ is even we deduce that we do not have a factor corresponding to an odd cycle, and we obtain the inequalities (2.12) and (2.14). Assume that $n$ is odd. Use (2.11) to deduce

$$
\begin{aligned}
q_{3} q_{4} \prec q_{7}, q_{3} q_{6} \prec q_{9} \prec q_{4} q_{5}, q_{5} q_{6} & \prec q_{11} \prec q_{4} q_{7}, \\
q_{4}^{2} q_{5}=q_{4}\left(q_{9}+x^{4}\right)=q_{13}+x^{4} q_{5}+x^{4} q_{4} & \succ q_{13}+x^{6}=q_{6} q_{7} .
\end{aligned}
$$

These inequalities yield (2.13) and (2.15). Equality in (2.12-2.15) if and only if we did not apply Theorem 2.1 at all.

We discuss second the lower bounds on $\Phi_{G}$. If $l \geq 6$ then we use the inequality $q_{l} \succ q_{3} q_{l-3}$. Use repeatedly this inequality, until we replaced the products of different $q_{l}$ with products involving $q_{3}, q_{4}$ and $q_{5}$. As

$$
q_{4}^{2} \succ q_{8} \succ q_{3} q_{5}, \quad q_{4} q_{5} \succ q_{9} \succ q_{3}^{3}, \quad q_{5}^{2}=q_{10}-2 x^{5}=q_{3} q_{7}+x^{3} q_{4}-2 x^{5} \succ q_{3} q_{7} \succ q_{3}^{2} q_{4},
$$

we deduce (2.16-2.18). Equalities hold if we did not apply Theorem 2.1 at all.

Assume finally that $G$ is a 2-regular bipartite multigraph on $n$ vertices. Then $G$ is a union of even cycles $C_{2 i}$ for $i \in \mathbb{N}$. Assume that $C_{i}$ and $C_{j}$ are even cycles. Then Theorem 2.1 yields that $q_{i} q_{j} \succ q_{i+j}$. Continue this process until we deduce that $\Phi_{G} \succeq q_{n}$. Equality holds if and only if $G=C_{n}$.

Use Theorem 2.2 and Theorem 2.1 for $i=2$ to deduce.

\section{Corollary 2.3}

- Let $G$ be a simple 2-regular graph on $4 q$ vertices. Then $\Phi_{G} \preceq \Phi_{q K_{2,2}}$. Equality holds if and only if $G=q K_{2,2}$.

- Let $G$ be a 2-regular multigraph on $2 n$ vertices. Then $\Phi_{G} \preceq \Phi_{n H_{2}}$. Equality holds if and only if $\mathrm{G}=\mathrm{nH}_{2}$.

Note that the above results verify all the claims we stated about 2-regular bipartite graphs in the Introduction.

\section{Graphs of degree at most 2}

Denote by $\Omega(n, k) \subset \Omega_{\text {mult }}(n, k)$ the set of simple graphs and multigraphs on $n$ vertices respectively, which have $2 k$ vertices, $(k>0)$, of degree 1 and the remaining vertices have degree 2. The following proposition is straightforward. 


\section{Proposition 3.1}

- Each $G \in \Omega(n, k)$ is a union of $k$ paths and possibly cycles $C_{i}$ for $i \geq 3$.

- Each $G \in \Omega_{\text {mult }}(n, k)$ is a union of $k$ paths and possibly cycles $C_{i}$ for $i \geq 2$.

$\Omega_{\text {mult }}(n, k) \backslash \Omega(n, k) \neq \emptyset$ if and only if $n-2 k \geq 2$.

Denote by $\Pi(n, k) \subseteq \Omega(n, k)$ the subset of graphs $G$ on $n$ vertices which are union of $k$-paths. Note that $\Pi(2 k, k)=k P_{2}$. As in $\S 2$ we study the minimum and maximum $m$-matchings in $\Pi(n, k), \Omega(n, k), \Omega_{\text {mult }}(n, k)$.

We first study the case where $G \in \Pi(n, 4)$, i.e. $G$ is a union of two paths with the total number of vertices equal to $n$.

Lemma 3.2 Let $n \geq 4$. Then

- If $n=0,1 \bmod 4$ then

$$
\begin{aligned}
p_{n-1} & =p_{1} p_{n-1} \prec p_{3} p_{n-3} \prec \cdots \prec p_{2\left\lfloor\frac{n}{4}\right\rfloor-1} p_{n-2\left\lfloor\frac{n}{4}\right\rfloor+1} \\
& \prec p_{2\left\lfloor\frac{n}{4}\right\rfloor} p_{n-2\left\lfloor\frac{n}{4}\right\rfloor} \prec p_{2\left\lfloor\frac{n}{4}\right\rfloor-2} p_{n-2\left\lfloor\frac{n}{4}\right\rfloor+2} \prec \cdots \prec p_{2} p_{n-2} \prec p_{0} p_{n}=p_{n} .
\end{aligned}
$$

- If $n=2,3 \bmod 4$ then

$$
\begin{aligned}
p_{n-1} & =p_{1} p_{n-1} \prec p_{3} p_{n-3} \prec \cdots \prec p_{2\left\lfloor\frac{n}{4}\right\rfloor+1} p_{n-2\left\lfloor\frac{n}{4}\right\rfloor-1} \\
& \prec p_{2\left\lfloor\frac{n}{4}\right\rfloor} p_{n-2\left\lfloor\frac{n}{4}\right\rfloor} \prec p_{2\left\lfloor\frac{n}{4}\right\rfloor-2} p_{n-2\left\lfloor\frac{n}{4}\right\rfloor+2} \prec \cdots \prec p_{2} p_{n-2} \prec p_{0} p_{n}=p_{n} .
\end{aligned}
$$

Proof. Let $0 \leq i, j$ and consider the path $P_{i+j}$. By considering the generating matching polynomial without the match $(i, i+1)$ and with match $(i, i+1)$ we get the identity

$$
p_{i+j}=p_{i} p_{j}+x p_{i-1} p_{j-1}
$$

Hence $p_{i+j}=p_{i-1} p_{j+1}+x p_{i-2} p_{j}$. Subtracting from this equation (3.3) we obtain $p_{i-1} p_{j+1}-$ $p_{i} p_{j}=-x\left(p_{i-2} p_{j}-p_{i-1} p_{j-1}\right)$. Assume that $i \leq j-2$. Continuing this process $i-1$ times, and taking in account that $p_{-1}=0, p_{-2}=\frac{1}{x}$ we get

$$
p_{i-1} p_{j+1}-p_{i} p_{j}=(-1)^{i-1} x^{i} p_{j-i} \text { for } 0 \leq i \leq j-2 .
$$

Hence $p_{i-2} p_{j+2}-p_{i-1} p_{j+1}=(-1)^{i-2} x^{i-1} p_{j-i+2}$. Add this equation to the previous one and use (2.5) to obtain

$$
p_{i-2} p_{j+2}-p_{i} p_{j}=(-1)^{i-2} x^{i-1} p_{j-i+1} \text { for } 1 \leq i \leq j-2 .
$$

We now prove (3.1-3.2). In (3.5) assume that $i \geq 3$ is odd and $j \geq i$. So $(-1)^{i-2}=-1$. Hence $p_{i-2} p_{j+2}-p_{i} p_{j} \prec 0$. This explains the ordering of the polynomials appearing in the first line of (3.1-3.2). Assume now that $i \geq 2$ is even and $j \geq i$. So $(-1)^{i-2}=1$. Hence $p_{i-2} p_{j+2}-p_{i} p_{j} \succ 0$. This explains the ordering of the polynomials appearing in the second line of (3.1-3.2).

The last inequality in the first line of (3.1-3.2) is implied by (3.4). 
Theorem 3.3 Let $k \geq 2, n \geq 2 k$. Then for any $G \in \Pi(n, k)$

$$
\Phi_{J} \preceq \Phi_{G} \preceq \Phi_{K} .
$$

Equality in the left-hand side and right-hand side holds if and only if $G=J$ and $G=K$ respectively. Here $K=(k-1) P_{2} \cup P_{n-2 k+2}$ and $J$ is defined as follows:

1. If $n \leq 3 k$ then $J=(3 k-n) P_{2} \cup(n-2 k) P_{3}$.

2. If $n>3 k$ then $J=(k-1) P_{3} \cup P_{n-3 k+3}$.

Proof. For $k=2$ the theorem follows from Lemma 3.2. For $k>2$ apply the theorem for $k=2$ for any two paths in $G \in \Pi(n, k)$ to deduce that $K$ and $J$ are the maximal and the minimal graphs respectively.

We extend the result of Lemma 3.2 for cycles.

Lemma 3.4 Let $n \geq 4$. Then

- If $n=0,1 \bmod 4$ then

$$
\begin{aligned}
q_{n-1} & =q_{1} q_{n-1} \prec q_{3} q_{n-3} \prec \cdots \prec q_{2\left\lfloor\frac{n}{4}\right\rfloor-1} q_{n-2\left\lfloor\frac{n}{4}\right\rfloor+1} \\
& \prec q_{2\left\lfloor\frac{n}{4}\right\rfloor} q_{n-2\left\lfloor\frac{n}{4}\right\rfloor} \prec q_{2\left\lfloor\frac{n}{4}\right\rfloor-2} q_{n-2\left\lfloor\frac{n}{4}\right\rfloor+2} \prec \cdots \prec q_{2} q_{n-2} \prec q_{n+1} .
\end{aligned}
$$

- If $n=2,3 \bmod 4$ then

$$
\begin{aligned}
q_{n-1} & =q_{1} q_{n-1} \prec q_{3} q_{n-3} \prec \cdots \prec q_{2\left\lfloor\frac{n}{4}\right\rfloor+1} q_{n-2\left\lfloor\frac{n}{4}\right\rfloor-1} \\
& \prec q_{2\left\lfloor\frac{n}{4}\right\rfloor} q_{n-2\left\lfloor\frac{n}{4}\right\rfloor} \prec q_{2\left\lfloor\frac{n}{4}\right\rfloor-2} q_{n-2\left\lfloor\frac{n}{4}\right\rfloor+2} \prec \cdots \prec q_{2} q_{n-2} \prec q_{n+1} .
\end{aligned}
$$

Proof. The equality (2.7) implies

$$
q_{n+1}=q_{n}+x q_{n-1}=q_{n-1}+x q_{n-2}+x q_{n-2}+x^{2} q_{n-3} \succ q_{n-2}+2 x q_{n-2}=q_{2} q_{n-2} .
$$

Hence the last inequality in (3.7) and (3.8) holds. By (2.11) we have $q_{i} q_{j}-q_{i+j}=$ $(-1)^{i} x^{i} q_{j-i}$. Using this, it is easy to see that

$$
q_{i-1} q_{j+1}-q_{i} q_{j}=(-1)^{i-1} x^{i-1} q_{j-i+2}-(-1)^{i} x^{i} q_{j-i}=(-1)^{i-1} x^{i-1}\left(q_{j-i+2}+x q_{j-i}\right),
$$

as well as

$$
\begin{aligned}
q_{i-2} q_{j+2}-q_{i} q_{j} & =(-1)^{i-2} x^{i-2} q_{j-i+4}-(-1)^{i} x^{i} q_{j-i} \\
& =(-1)^{i-2} x^{i-2}\left(q_{j-i+4}-x^{2} q_{j-i}\right) \\
& =(-1)^{i-2} x^{i-2}\left(q_{j-i+3}+x q_{j-i+2}-x^{2} q_{j-i}\right) \\
& =(-1)^{i-2} x^{i-2}\left(q_{j-i+3}+x q_{j-i+1}\right) .
\end{aligned}
$$

Comparing these equalities with (3.4) and (3.5) we obtain all other inequalities in (3.7) and (3.8).

Next, we study graphs composed of a path and a cycle of the form $p_{i} q_{j}$. 
Lemma 3.5 Let $n \geq 4$. Then

- If $n=0,1 \bmod 4$ then

$$
\begin{aligned}
q_{n-1} & =p_{1} q_{n-1} \prec q_{3} p_{n-3} \prec p_{3} q_{n-3} \prec q_{5} p_{n-5} \prec p_{5} q_{n-5} \prec \ldots \\
& \prec q_{2\left\lfloor\frac{n}{4}\right\rfloor-1} p_{n-2\left\lfloor\frac{n}{4}\right\rfloor+1} \prec p_{2\left\lfloor\frac{n}{4}\right\rfloor-1} q_{n-2\left\lfloor\frac{n}{4}\right\rfloor+1} \prec p_{2\left\lfloor\frac{n}{4}\right\rfloor} q_{n-2\left\lfloor\frac{n}{4}\right\rfloor} \preceq q_{2\left\lfloor\frac{n}{4}\right\rfloor} p_{n-2\left\lfloor\frac{n}{4}\right\rfloor} \\
& \prec p_{2\left\lfloor\frac{n}{4}\right\rfloor-2} q_{n-2\left\lfloor\frac{n}{4}\right\rfloor+2} \prec q_{2\left\lfloor\frac{n}{4}\right\rfloor-2} p_{n-2\left\lfloor\frac{n}{4}\right\rfloor+2} \prec \ldots \\
& \prec p_{4} q_{n-4} \prec q_{4} p_{n-4} \prec p_{2} q_{n-2} \prec q_{2} p_{n-2} \prec p_{0} q_{n}=q_{n} .
\end{aligned}
$$

(If $n=0 \bmod 4$ then $\preceq$ is $=$, and otherwise $\preceq$ is $\prec$.)

- If $n=2,3 \bmod 4$ then

$$
\begin{aligned}
q_{n-1} & =p_{1} q_{n-1} \prec q_{3} p_{n-3} \prec p_{3} q_{n-3} \prec \cdots \prec q_{2\left\lfloor\frac{n}{4}\right\rfloor+1} p_{n-2\left\lfloor\frac{n}{4}\right\rfloor-1} \\
& \preceq p_{2\left\lfloor\frac{n}{4}\right\rfloor+1} q_{n-2\left\lfloor\frac{n}{4}\right\rfloor-1} \prec p_{2\left\lfloor\frac{n}{4}\right\rfloor} q_{n-2\left\lfloor\frac{n}{4}\right\rfloor} \prec q_{2\left\lfloor\frac{n}{4}\right\rfloor} p_{n-2\left\lfloor\frac{n}{4}\right\rfloor} \\
& \prec p_{2\left\lfloor\frac{n}{4}\right\rfloor-2} q_{n-2\left\lfloor\frac{n}{4}\right\rfloor+2} \prec q_{2\left\lfloor\frac{n}{4}\right\rfloor-2} p_{n-2\left\lfloor\frac{n}{4}\right\rfloor+2} \prec \ldots \\
& \prec p_{4} q_{n-4} \prec q_{4} p_{n-4} \prec p_{2} q_{n-2} \prec q_{2} p_{n-2} \prec p_{0} q_{n}=q_{n} .
\end{aligned}
$$

(If $n=2 \bmod 4$ then $\preceq$ is $=$, and otherwise $\preceq$ is $\prec$.)

Proof. Assume that $0 \leq i, 2 \leq j$. Use (2.6) to obtain

$$
p_{i} q_{j}-q_{i+2} p_{j-2}=p_{i}\left(p_{j}+x p_{j-2}\right)-\left(p_{i+2}+x p_{i}\right) p_{j-2}=p_{i} p_{j}-p_{i+2} p_{j-2}
$$

(3.5) implies

$$
\begin{aligned}
& p_{i} q_{j}-q_{i+2} p_{j-2}=(-1)^{i} x^{i+1} p_{j-i-3} \text { if } i \leq j-3, \\
& p_{i} q_{j}-q_{i+2} p_{j-2}=(-1)^{j-1} x^{j-1} p_{i-j+1} \text { if } i \geq j-2
\end{aligned}
$$

Assume that $0 \leq i \leq j-3$. Hence, if $i$ is odd we get that $p_{i} q_{j} \prec q_{i+2} p_{j-2}$. If $i$ is even then $q_{i+2} p_{j-2} \prec p_{i} q_{j}$. These inequalities yield slightly less than the half of the inequalities in (3.9) and (3.10).

Assume that $1 \leq i<j$. Use (2.6) and (3.5) to deduce

$$
p_{i} q_{j}-q_{i} p_{j}=p_{i} p_{j}-p_{i} p_{j}+x\left(p_{i} p_{j-2}-p_{i-2} p_{j}\right)=(-1)^{i-1} x^{i} p_{j-i-1}
$$

Therefore, if $i$ is odd then $q_{i} p_{j} \prec p_{i} q_{j}$. If $i$ is even then $p_{i} q_{j} \prec q_{i} p_{j}$. These inequalities yield slightly less than the other half of the inequalities in (3.9) and (3.10).

Assume that $0 \leq i \leq j$. Use (2.6) and (3.4) to deduce

$$
\begin{aligned}
p_{i-1} q_{j+1}-p_{i} q_{j} & =p_{i-1} p_{j+1}-p_{i} p_{j}+x\left(p_{i-1} p_{j-1}-p_{i} p_{j-2}\right) \\
& =(-1)^{i-1} x^{i}\left(p_{j-i}+x p_{j-i-2}\right)=(-1)^{i-1} x^{i} q_{j-i} .
\end{aligned}
$$


If $i$ is even then $p_{i-1} q_{j+1} \prec p_{i} q_{j}$. This shows the first inequality in the second line of (3.9). If $i$ is odd then $p_{i} q_{j} \prec p_{i-1} q_{j+1}$. This shows the inequality between the last term of the first line and the first term in the second line of (3.10).

For graphs consisting of more than two cycles or paths there is no total ordering by coefficients of matching polynomials. In particular, we computed that $p_{8} p_{6} p_{3}$ is not comparable with $p_{7} p_{5} p_{5}$. The same holds true for the same parameters with cycles instead of paths. To show that this is not due solely to the mixed parity of path/cycle length, we also showed that $p_{4} p_{4} p_{16} p_{28}$ is incomparable with $p_{6} p_{6} p_{6} p_{34}$.

To extend the results of Theorem 3.3 to graphs in $\Omega(n, k)$ we need the following lemma.

Lemma 3.6 Let $5 \leq i \in \mathbb{N}$. Then

$$
\begin{aligned}
p_{i}-q_{3} p_{i-3} & =x^{2} p_{i-6}, \\
p_{i}-p_{2} q_{i-2} & =-x^{3} p_{i-6}, \\
p_{i+1}-p_{3} q_{i-2} & =x^{4} p_{i-7} . \\
p_{2 i-3}-q_{4} p_{2 i-7} & =-x^{4} p_{2 i-11} .
\end{aligned}
$$

Hence

$$
\begin{aligned}
& \Phi_{P_{5}}=\Phi_{C_{3} \cup P_{2}}, \quad \Phi_{P_{7}}=\Phi_{P_{3} \cup C_{4}}, \text { and } \Phi_{P_{i}} \succ \Phi_{C_{3} \cup P_{i-3}}, \\
& \Phi_{P_{i}} \prec \Phi_{P_{2} \cup C_{i-2}}, \quad \Phi_{P_{i+2}} \succ \Phi_{P_{3} \cup C_{i-1}}, \quad \Phi_{P_{2 i-3}} \prec \Phi_{P_{2 i-7} \cup C_{4}} \text { for } i \geq 6 .
\end{aligned}
$$

Furthermore,

$$
p_{2 i+2 j} \prec p_{2 i} q_{2 j} \text { for any nonnegative integers } i, j \text {. }
$$

In particular, $\Phi_{P_{2 i+2 j}} \prec \Phi_{P_{2 i} \cup C_{2 j}}$ for $i, j \in \mathbb{N}$.

Proof. Use (2.7) and (3.4-3.5) to obtain

$$
\begin{aligned}
p_{i}-q_{3} p_{i-3} & =p_{0} p_{i}-p_{2} p_{i-2}+p_{2} p_{i-2}-p_{3} p_{i-3}-x p_{i-3}=x p_{i-3}+x^{2} p_{i-6}-x p_{i-3} \\
& =x^{2} p_{i-6}, \\
p_{i}-p_{2} q_{i-2} & =p_{0} p_{i}-p_{2} p_{i-2}-x p_{2} p_{i-4}=x\left(p_{1} p_{i-3}-p_{2} p_{i-4}\right)=-x^{3} p_{i-6}, \\
p_{i+1}-p_{3} q_{i-2} & =p_{0} p_{i+1}-p_{2} p_{i-1}+p_{2} p_{i-1}-p_{3} p_{i-2}-x p_{3} p_{i-4}=x p_{i-2}+x^{3} p_{i-5}-x p_{3} p_{i-4} \\
& =x\left(p_{1} p_{i-2}-p_{3} p_{i-4}\right)+x^{3} p_{i-5}=x^{3}\left(p_{i-5}-p_{i-6}\right)=x^{4} p_{i-7}, \\
p_{2 i-3}-q_{4} p_{2 i-7} & =p_{0} p_{2 i-3}-p_{4} p_{2 i-7}-x p_{2} p_{2 i-7} \\
& =\left(p_{0} p_{2 i-3}-p_{2} p_{2 i-5}\right)+\left(p_{2} p_{2 i-5}-p_{4} p_{2 i-7}\right)-x p_{2} p_{2 i-7} \\
& =x p_{2 i-6}+x^{3} p_{2 i-10}-x p_{2} p_{2 i-7}=x\left(p_{1} p_{2 i-6}-p_{2} p_{2 i-7}\right)+x^{3} p_{2 i-10} \\
& =-x^{3} p_{2 i-9}+x^{3} p_{2 i-10}=-x^{4} p_{2 i-11} .
\end{aligned}
$$

These equalities imply (3.15-3.18). Recall that $p_{-1}=0, p_{0}=p_{1}=1$ and $p_{i} \succ 0$ for $i \geq 0$ to deduce the implications of the above identities. 
To prove (3.19) recall that $p_{0}=1, q_{0}=2, q_{i} \succ 0$. Hence it is enough to consider the cases $i, j \geq 1$. In view of Lemma 3.5 it is enough to assume that $1 \leq i \leq j \leq i+1$. Use (2.6) and (3.3) to obtain

$$
p_{2 i} q_{2 j}-p_{2 i+2 j}=x p_{2 i} p_{2 j-2}-x p_{2 i-1} p_{2 j-1}=-x\left(p_{2 i-1} p_{2 j-1}-p_{2 i} p_{2 j-2}\right) .
$$

Use (3.4) and the equalities $p_{0}=1, p_{2}=\frac{1}{x}$ to obtain

$$
p_{2 i} q_{2 j}-p_{2 i+2 j}=x^{2 i+1} p_{2 j-2 i-2} \succ 0 .
$$

Theorem 3.7 Let $G$ be a simple graph of order $n$ with degree sequence $d_{1}=\cdots=$ $d_{2 k}=1$ and $d_{2 k+1}=\cdots=d_{n}=2,2 \leq 2 k \leq n$, i.e. $G \in \Omega(n, k)$. Set $n-2 k=l$ and assume that $l \geq 2$. (Otherwise $\Omega(n, k)$ consists of one graph.) Then

$$
\Phi_{F} \preceq \Phi_{G} \preceq \Phi_{H},
$$

where the graphs $F$ and $H$ depend on $n$ and $k$ as follows.

1. When $l-k \leq 0$ then $F=l P_{3} \cup(k-l) P_{2}$.

2. When $l-k>0$

(a) If $l-k \equiv 0(\bmod 3)$, then $F=k P_{3} \cup \frac{1}{3}(l-k) C_{3}$.

(b) If $l-k \equiv 1(\bmod 3)$, then $F=(k-1) P_{3} \cup P_{4} \cup \frac{1}{3}(l-k-1) C_{3}$.

(c) If $l-k \equiv 2(\bmod 3)$, then either $F=F_{1}=(k-1) P_{3} \cup P_{5} \cup \frac{1}{3}(l-k-2) C_{3}$ or $F=F_{2}=(k-1) P_{3} \cup P_{2} \cup \frac{1}{3}(l-k+1) C_{3}$.

3. If $l=2$ then $H=(k-1) P_{2} \cup P_{4}$.

4. If $l=3$ then either $H=(k-1) P_{2} \cup P_{5}$ or $H=k P_{2} \cup C_{3}$.

5. If $l \geq 4$ and $l \equiv 0(\bmod 4)$, then $H=k P_{2} \cup \frac{1}{4} l C_{4}$.

6. If $l \geq 5$ and $l \equiv 1(\bmod 4)$, then $H=k P_{2} \cup \frac{1}{4}(l-5) C_{4} \cup C_{5}$.

7. If $l \geq 6$ and $l \equiv 2(\bmod 4)$, then $H=k P_{2} \cup \frac{1}{4}(l-6) C_{4} \cup C_{6}$.

8. If $l \geq 7$ and $l \equiv 3(\bmod 4)$, then $H=k P_{2} \cup \frac{1}{4}(l-7) C_{4} \cup C_{7}$.

Furthermore, if $G \neq F$ then $\Phi_{F} \prec \Phi_{G}$ and if $G \neq H$ then $\Phi_{G} \prec \Phi_{H}$. 
Proof. Consider a partial order on $\Omega(n, k)$ induced by the partial order $\preceq$ on $\mathbb{R}_{+}[x]$. Thus $G_{1} \ll G_{2} \Longleftrightarrow \Phi_{G_{1}} \preceq \Phi_{G_{2}}$. It is enough to show that any minimal and maximal element in $\Omega(n, k)$ with respect to this order is of the form $F$ and $H$ respectively.

Assume that $G$ is a minimal element with respect to this partial order. Hence there is no $G^{\prime} \in \Omega(n, k)$ such that $\Phi_{G^{\prime}} \prec \Phi_{G}$. Suppose that $G$ has at least one cycle. Theorem 2.2 implies that $G$ contains at most one cycle $C_{i} \neq C_{3}$, where $i \in[4,5]$. We now rule out such $C_{i}$. Since $k \geq 1 G$ must contain a path $P_{j}$ for $j \geq 2$. Lemma 3.5 yields that $q_{3} p_{i+j-3} \prec p_{j} q_{i}$. Hence if we replace $C_{i} \cup P_{j}$ with $C_{3} \cup P_{i+j-3}$ we will obtain $G^{\prime} \in \Omega(n, k)$ such that $\Phi_{G^{\prime}} \prec \Phi_{G}$. This contradicts the minimality of $G$. Hence $G$ can contain only cycles of length 3 .

In view of Lemma $3.6 G$ does not contain $P_{i}$ with $i \geq 6$. Denote by $\mathcal{B}_{2}, \mathcal{B}_{3}$ and $\mathcal{B}_{4}$ the set of paths with 2, 3 and at least 4 vertices in $G$ respectively. We claim that $\# \mathcal{B}_{4} \leq 1$. Otherwise, let $Q, R \in \mathcal{B}_{4}$ be two different paths. Lemma 3.2 yields that $\Phi_{P_{3} \cup P_{i-1}} \prec \Phi_{Q \cup R}$. This contradicts the minimality of $G$. Next we observe that that $\min \left(\# \mathcal{B}_{2}, \# \mathcal{B}_{4}\right)=0$. If not, choose $Q \in \mathcal{B}_{2}, R \in \mathcal{B}_{4}$. Lemma 3.2 yields that $\Phi_{P_{3} \cup P_{i-1}} \prec \Phi_{Q \cup R}$, which contradicts the minimality of $G$.

We claim that $G$ has to be of the form $F$. Suppose first that $G$ does not have cycles. If $\mathcal{B}_{4}=\emptyset$ then we are in the case 1 . If $\mathcal{B}_{2}=\emptyset$ then we have either the case $2 b$ with $l=k+1$ or the case $2 c$ with $l=k+2$ and $F=F_{1}$.

Assume now that $G$ has cycles. If $\mathcal{B}_{2}=\mathcal{B}_{4}=\emptyset$ then we have the case 2a. Assume now that $\mathcal{B}_{2}=\emptyset$ and $\# \mathcal{B}_{4}=1$. Then we have either the case $2 b$ with $l>k+1$ or the case $2 c$ with $l>k+2$ and $F=F_{1}$.

Assume finally that $\mathcal{B}_{4}=\emptyset$ and $\# \mathcal{B}_{2} \geq 1$. We claim that $\# \mathcal{B}_{2}=1$. Assume to the contrary that $\mathcal{B}_{2}$ contains at least two $P_{2}$. Since $G$ contains at least one cycle $C_{3}$ we replace $P_{2} \cup C_{3}$ with $P_{5}$ to obtain another minimal $G^{\prime}$. As $G^{\prime}$ contains $P_{2}$ and $P_{5}$ it is not minimal, contrary to our assumption. Hence $\# \mathcal{B}_{2}=1$ and we have the case $2 c$ and $G=F_{2}$.

We now assume that $G$ is a maximal element in $\Omega(n, k)$. Thus, there is no $G^{\prime} \in \Omega(n, k)$ such that $\Phi_{G} \prec \Phi_{G^{\prime}}$.

Observe first $G$ does not contain two distinct paths $Q, R$ with $i, j \geq 3$ vertices. Indeed, Lemma 3.2 implies that $\Phi_{Q \cup R} \prec \Phi_{P_{2} \cup P_{i+j-2}}$. This shows that $G=H$ in the cases 3 and 4. (In the case 4 we use the identity $\Phi_{P_{5}}=\Phi_{P_{2} \cup C_{3}}$.)

In what follows we assume that $l \geq 4$. Observe next that $G$ cannot contain $P_{i}$, where $i \geq 6$. Otherwise replace $P_{i}$ with $P_{2} \cup C_{i-2}$ and use (3.16).

Also $G$ cannot contain a cycle $C_{i}, i \geq 3$ and a path $P_{j}$ for $j \geq 3$. Indeed, in view of Lemma 3.5 we have the inequality $\Phi_{P_{j} \cup C_{i}} \prec \Phi_{P_{2} \cup C_{i+j-2}}$.

Since $l \geq 4$ it follows that $G$ has at least one cycle and all paths in $G$ are of length 2. Theorem 2.2 implies that $G$ contains at most one cycle $C_{i} \neq C_{4}$, where $i \in[5,6,7]$. It now follows that $G=H$, where $H$ satisfies one of the conditions 5-8.

We now a give the version of Theorem 3.7 for the subset $\Omega_{\mathrm{bi}}(n, k) \subset \Omega(n, k)$ of bipartite graphs. 
Theorem 3.8 Let $G$ be a simple bipartite graph of order $n$ with degree sequence $d_{1}=$ $\cdots=d_{2 k}=1$ and $d_{2 k+1}=\cdots=d_{n}=2$, where $2 \leq 2 k \leq n$, i.e. $G \in \Omega_{b i}(n, k)$. Set $n-2 k=l$, and assume that $l \geq 2$. Then (3.20) holds, where the graphs $F$ and $H$ depend on $n$ and $k$ as follows.

1. When $l-k \leq 0$ then $F=l P_{3} \cup(k-l) P_{2}$.

2. When $l-k>0$

(a) If $l-k=1,2$ then $F=(k-1) P_{3} \cup P_{l-k+3}$.

(b) If $4 \leq l-k$ even then either $F=F_{1}=k P_{3} \cup C_{l-k}$ or if $l-k=4$ then $F=F_{2}=(k-1) P_{3} \cup P_{7}$.

(c) If $3 \leq l-k$ is odd, then $F=(k-1) P_{3} \cup P_{l-k+3}$.

3. If $l=2$ then $H=(k-1) P_{2} \cup P_{4}$.

4. If $l=3$ then $H=(k-1) P_{2} \cup P_{5}$.

5. If $l \geq 4$ and $l \equiv 0(\bmod 4)$, then $H=k P_{2} \cup \frac{1}{4} l C_{4}$.

6. If $l \geq 5$ and $l \equiv 1(\bmod 4)$, then $H=H_{1}=(k-1) P_{2} \cup \frac{1}{4}(l-1) C_{4} \cup P_{3}$ or $H=H_{2}=(k-1) P_{2} \cup \frac{1}{4}(l-5) C_{4} \cup P_{7}$.

7. If $l \geq 6$ and $l \equiv 2(\bmod 4)$, then $H=k P_{2} \cup \frac{1}{4}(l-6) C_{4} \cup C_{6}$.

8. If $l \geq 7$ and $l \equiv 3(\bmod 4)$, then $H=H_{1}=(k-1) P_{2} \cup \frac{1}{4}(l-3) C_{4} \cup P_{5}$.

Furthermore, if $G \neq F$ then $\Phi_{F} \prec \Phi_{G}$ and if $G \neq H$ then $\Phi_{G} \prec \Phi_{H}$.

Proof. The proof of this theorem is similar to the proof of Theorem 3.7, and we briefly point out the different arguments one should make. First, recall that $G \in \Omega(n, k)$ is bipartite, if and only if $G$ contains only even cycles.

We first assume that $G$ is minimal. Lemma 3.2 implies that $G$ cannot contain two paths, such that either each at least length 4 , or one of length 2 and one of length at least 4. Use (3.17) to deduce that $G$ cannot contain $P_{i}$ for $i \geq 9$. Also note that $\Phi_{P_{7}}=\Phi_{P_{3} \cup C_{4}}$. By Theorem 2.2 $G$ can contain at most one even cycle. Furthermore (3.19) yields that $G$ cannot contain an even cycle and an even path. This show that the minimal $G$ must be equal to $F$.

Assume now that $G$ is maximal. Note that in view of Theorem 3.7 we need only to consider the cases 6 and 8 , i.e. $l \geq 5, l \equiv 1 \bmod 4$ and $l \geq 7, l \equiv 3 \bmod 4$.

In view of Theorem 2.2 can have at most one cycle of length 6 , while all the other are of length 4. Lemma 3.2 implies that one out of any two paths in $G$ is $P_{2}$. (3.16) implies that $G$ does not contain an even path of length greater than 5. Lemma 3.5 implies that if $G$ contains an even path and a cycle then the length of the even path is 2. (3.18) yields that $G$ does not contain an odd path of length greater than 8. Also one has the equality $\Phi_{P_{7}}=\Phi_{P_{3} \cup C_{4}}$ (Lemma 3.6). 
Thus, if an odd path appears in $G$ then we may assume it is one of the following: $P_{3}$, $P_{5}$ or $P_{7}$. First we compare $p_{3} q_{6}$ with $p_{5} q_{4}$. (3.9) yields $p_{3} q_{6} \prec q_{4} p_{5}$. This establishes the case 8 . Next we compare $p_{7} q_{4}$ with $p_{5} q_{6}$. Use (3.11) to obtain $p_{4} q_{7}-q_{6} p_{5}=x^{5}$. Next use (3.13) to show that $p_{4} q_{7}-q_{4} p_{7}=-x^{4} p_{2}$. Hence $q_{4} p_{7}-q_{6} p_{5}=x^{4} p_{2}+x^{5}$. Hence $\Phi_{P_{7} \cup C_{4}} \succ \Phi_{P_{5} \cup C_{6}}$. This establishes 6 .

\section{Expected values of number of $m$-matchings}

\subsection{First measure}

For a set $\mathcal{A} \subset \mathbb{R}$ denote by $\mathcal{A}^{p \times q}$ the set of $p \times q$ matrices $A=\left[a_{i j}\right]_{i, j=1}^{p, q}$, where each entry $a_{i j}$ is in $\mathcal{A}$. For $A=\left[a_{i j}\right] \in \mathbb{R}^{n \times n}$ denote by perm $A$ the permanent of $A$, i.e. $\operatorname{perm} A=\sum_{\sigma \in \mathrm{S}_{\mathrm{n}}} \prod_{i=1}^{n} a_{i \sigma(i)}$, where $\mathrm{S}_{\mathrm{n}}$ is the permutation group on $\langle n\rangle$. Let $A \in \mathbb{R}^{p \times q}$ and $m \in\langle\min (p, q)\rangle$. Denote by $\operatorname{perm}_{m} A$ the sum of permanents of all $m \times m$ submatrices of $A$.

Denote by $\mathcal{G}(p, q)$ and $\mathcal{G}_{\text {mult }}(p, q)$ the set of simple bipartite graphs and bipartite multigraphs on $p$ and $q$ vertices in each class, respectively. W.L.O.G., we can assume that $1 \leq p \leq q$. We identify the two classes $p$ and $q$ vertices with $\langle p\rangle$ and $\langle q\rangle$. (Sometimes we identify the second class with $q$ vertices with $\langle q\rangle+p:=\{p+1, \ldots, p+q\}$.) For $G \in \mathcal{G}(p, q)$ let $A(G)=\left[a_{i j}\right]_{i, j=1}^{p, q} \in\{0,1\}^{p \times q}$ be the $(0,1)$ matrix representing $G$. Vice versa, any $A \in\{0,1\}^{p \times q}$ represents a unique graph $G \in \mathcal{G}(p, q)$. Let $G_{1}, \ldots, G_{r} \in \mathcal{G}(p, q)$. Let $G$ be a bipartite multigraph on the vertices $\langle p\rangle \cup\langle q\rangle$, whose set of edges is the union of the set of edges in $G_{i}$. I.e., $e \in\langle p\rangle \times\langle q\rangle$, appears $l$ times in $G$, if and only exactly $l$ graphs from $G_{1}, \ldots, G_{r}$ contain the edge $e$. We denote $G$ by $\vee_{i=1}^{r} G_{i}$. So $A(G)=\left[a_{i j}\right]=\sum_{i=1}^{r} A\left(G_{i}\right) \in\langle r\rangle^{p \times q}$. Vice versa, any $A \in\langle r\rangle^{p \times q}$ corresponds to a bipartite multigraph $G$ on the vertices $\langle p\rangle,\langle q\rangle$, such that $G=\vee_{i=1}^{r} G_{i}$, where $G_{i} \in \mathcal{G}(p, q)$. (Usually there would be many such decompositions of $G$.)

In what follows we need the following lemma.

Lemma 4.1 Let $p, q, r \in \mathbb{N}$ and assume that $G_{1}, \ldots, G_{r} \in \mathcal{G}(p, q)$. Let $A_{i}:=A\left(G_{i}\right) \in$ $\{0,1\}^{p \times q}$, and denote $A:=\sum_{i=1}^{r} A_{i}$. Let $m \in\langle\min (p, q)\rangle$. Then $\operatorname{perm}_{m} A$ is the number of $m$-matchings of $G:=\vee_{i=1}^{r} G_{i}$, which is equal to the number of $m$-matchings obtained in the following way. Consider $m_{1}, \ldots, m_{r} \in \mathbb{Z}_{+}$such that $m_{1}+\ldots+m_{r}=m$. In each $G_{i}$ choose an $m_{i}$-matching $M_{i}$ such that $\cup_{i=1}^{r} M_{i}$ is an m-matching, i.e., $M_{i} \cap M_{j}=\emptyset$ for each $i \neq j$.

Proof. Notice that $A$ is the incidence matrix for the multigraph $G:=\vee_{i=1}^{r} G_{i}$. The permanent of the incidence matrix of a multigraph can be viewed as the number of $m$-matchings of the same graph with multiple edges merged and each edge chosen as many times as its multiplicity but not in the same $m$-matching. 
Let $\mathcal{S}_{n}$ be the set of all $n \times n$ permutation matrices and set

$$
\mathcal{S}_{n}^{r}=\mathcal{S}_{n} \times \cdots \times \mathcal{S}_{n}:=\left\{\left(P_{1}, \ldots, P_{r}\right): P_{1}, \ldots, P_{r} \in \mathcal{S}_{n}\right\}
$$

Denote by $\mathcal{G}(2 n, r) \subset \mathcal{G}_{\text {mult }}(2 n, r)$ the set of simple and bipartite multigraphs on $\langle n\rangle,\langle n\rangle$ vertices, where each vertex has degree $r$. Denote by $\Delta(n, r) \subset\{0,1, \ldots, r\}^{n \times n}$ the set of matrices with nonnegative integer entries such that the sum of each row and column of $A$ is equal to $r$. That is each $A \in \Delta(n, r)$ is the incidence matrix of $G \in \mathcal{G}_{\text {mult }}(2 n, r)$. $G$ is simple if and only if $A \in\{0,1\}^{n \times n}$. Birkhoff-König theorem implies that each $A \in \Delta(n, r)$ is a sum of $r$-permutation matrices.

$$
A=P_{1}+\cdots+P_{r}, \quad P_{1}, \ldots, P_{r} \in \mathcal{S}_{n}
$$

Let $\phi: \mathcal{S}_{n}^{r} \rightarrow \Delta(n, r)$ is given by (4.1). Then for $A \in \Delta(n, r) \phi^{-1}(A)$ is the set of all $r$ tuples $\left(P_{1}, \ldots, P_{r}\right)$ which present $A$. Let $\# \phi^{-1}(A)$ be the cardinality of the set $\phi^{-1}(A)$.

View $\mathcal{S}_{n}^{r}$ as a discrete probability space where each point $\left(P_{1}, \ldots, P_{r}\right)$ has the equal probability $(n !)^{-r}$. Then $\phi: \mathcal{S}_{n}^{r} \rightarrow \Delta(n, r)$ induces the following probability measure on $\Delta(n, r)$ :

$$
P\left(X_{n, r}=A \in \Delta(n, r)\right)=\frac{\# \phi^{-1}(A)}{(n !)^{r}} .
$$

Here $X_{n, r}$ is a random variable on the set $\Delta(n, r)$.

Lemma 4.2 Let $1 \leq r \in N, 1 \leq m \leq n \in N$. Assume that the random variable $X_{n, r} \in \Delta(n, r)$ has the distribution given by (4.2). Then

$$
\begin{aligned}
& E_{1}(m, n, r):=E\left(\operatorname{perm}_{m} X_{n, r}\right)= \\
& \frac{1}{(n !)^{r}}\left(\begin{array}{c}
n \\
m
\end{array}\right)^{2} m ! \sum_{m_{1}, \ldots, m_{r} \in \mathbb{Z}_{+}, m_{1}+\cdots m_{r}=m} \frac{m !\left(n-m_{1}\right) ! \cdots\left(n-m_{r}\right) !}{m_{1} ! \cdots m_{r} !} .
\end{aligned}
$$

Proof. We first observe the following equality:

$$
\sum_{P_{1}, \ldots, P_{r} \in \mathcal{S}_{n}} P_{1}+\ldots+P_{r}=\sum_{A \in \Delta(n, r)}\left(\# \phi^{-1}(A)\right) A .
$$

(Just group $P_{1}+\ldots+P_{r}$ to $A \in \Delta(n, r)$.) Hence

$$
E\left(\operatorname{perm}_{m} X_{n, r}\right)=\frac{1}{(n !)^{r}} \sum_{P_{1}, \ldots, P_{r} \in \mathcal{S}_{n}} \operatorname{perm}_{m}\left(P_{1}+\ldots+P_{r}\right)
$$

We now compute the right-hand side of (4.4). Each $A=P_{1}+\ldots+P_{r}$ we interpret as a regular $r$-multigraph $G:=\vee_{i=1}^{r} G_{i}$. So $\operatorname{perm}_{m} A$ is the number of total $m$-matchings of $G$. It is given by Lemma 4.1. We now consider in the right-hand side of (4.4) all terms which contribute to a matching $(1, n+1), \ldots,(m, n+m)$. (Here $V_{1}=\{1, \ldots, n\}, V_{2}=$ $\{n+1, \ldots, 2 n\})$. 
To achieve that we choose an $r$ partition $U_{1}, \ldots, U_{r}$ of the set $\{1, \ldots, m\}$, so that $U_{i}$ has $m_{i} \geq 0$ elements. So $m_{1}+\cdots+m_{r}=m$. The choice of all such $U_{1}, \ldots, U_{r}$ is $\frac{m !}{m_{1} ! \cdots m_{r} !}$. Now once we choose $U_{i}$, it means that we assumed that we choose the edges $(j, n+j), j \in U_{i}$ from the graph $G_{i}$ for $i=1, \ldots, r$. This is possible if and only if $P_{i}$ fixes the elements of $U_{i}$. Then there are exactly $\left(n-m_{i}\right)$ ! permutations $P_{i}$ each of which fixes $U_{i}$. This gives the summand inside the summation in the right-hand side of (4.3). Next observe that after we decided that the $m$-matches are chosen from the sets $\{1, \ldots, m\} \times\{n+1, \ldots, n+m\}$ then the total possible set of $m$-matches for this choice is $m$ !. This gives the $m$ ! factor outside the summation in the right-hand side of (4.3). In general we should choose two subsets of size $m$ from $V_{1}$ and $V_{2}$. This gives the factor $\left(\begin{array}{l}n \\ m\end{array}\right)^{2}$. Finally the factor $\frac{1}{(n !)^{r}}$ is the probability of choosing $r$-tuple $\left(P_{1}, \ldots, P_{r}\right)$.

Lemma 4.3 Let $2 \leq r \leq m$ be integers. Let $\mu_{1}, \ldots, \mu_{r}$ be $r$ unique integers satisfying the conditions

$$
\mu_{i}=\left\lfloor\frac{m}{r}\right\rfloor, i=1, \ldots, k<r, \mu_{i}=\left\lceil\frac{m}{r}\right\rceil, i=k+1, \ldots, r, \sum_{i=1}^{r} \mu_{i}=m .
$$

Then

$$
\begin{aligned}
&\left(\begin{array}{c}
m+r-1 \\
r-1
\end{array}\right) \frac{1}{(n !)^{r-2}((n-m) !)^{2}} \prod_{i=1}^{r} \frac{\left(n-\mu_{i}\right) !}{\mu_{i} !} \geq \\
& E_{1}(m, n, r) \geq \frac{1}{(n !)^{r-2}((n-m) !)^{2}} \prod_{i=1}^{r} \frac{\left(n-\mu_{i}\right) !}{\mu_{i} !} .
\end{aligned}
$$

Proof. If $r$ divides $m$ then $\mu_{1}=\ldots=\mu_{r}=\frac{m}{r}$ and (4.5) trivially holds for any integer $k \in[1, r-1]$. Assume that $r$ does not divide. Then

$$
k=r\left\lceil\frac{m}{r}\right\rceil-m \text {. }
$$

Since the right-hand side of the inequality (4.6) is one of the nonnegative summands appearing in the definition (4.3) of $E_{1}(m, n, r)$ we immediately deduce the lower bound in (4.6).

We next claim the inequality

$$
\frac{\left(n-m_{1}\right) ! \cdots\left(n-m_{r}\right) !}{m_{1} ! \cdots m_{r} !} \leq \frac{\left(n-\mu_{1}\right) ! \cdots\left(n-\mu_{r}\right) !}{\mu_{1} ! \cdots \mu_{r} !}
$$

for any $r$ nonnegative integers such that $m_{1}+\ldots+m_{r}=m$. To show this inequality we start with the case $r=2$. Suppose that $0 \leq a<b-1$ and $a+b=m \leq n$. A straightforward calculation shows:

$$
\frac{(n-a) !(n-b) !}{a ! b !} \leq \frac{(n-(a+1)) !(n-(b-1)) !}{(a+1) !(b-1) !} .
$$


(Equality holds if and only if $a+b=n$.) Hence the maximum of the left-hand side of (4.8) on all possible nonnegative integers $m_{1}, \ldots, m_{r}$ whose sum is $m$ is achieved for $\left(m_{1}, \ldots, m_{r}\right)$ such that $\left|m_{i}-m_{j}\right| \leq 1$ for all $i \neq j$. This implies that the maximum of the left-hand side of (4.8) is achieved for any permutation of $\mu_{1}, \ldots, \mu_{r}$, which implies (4.8). It is well known that the number of nonnegative integers $m_{1}, \ldots, m_{r}$ which sum to $m$ is $\left(\begin{array}{c}m+r-1 \\ r-1\end{array}\right)$. Hence the equality (4.3) combined with (4.8) yields the upper bound in (4.6).

Theorem 4.4 Let $2 \leq r \in \mathbb{N}$. Assume that $1 \leq m_{k} \leq n_{k}, k=1, \ldots$, are two strictly increasing sequences of integers such that the sequence $\frac{m_{k}}{n_{k}}, k=1, \ldots$ converges to $p \in[0,1]$. Then

$$
\lim _{k \rightarrow \infty} \frac{\log E_{1}\left(m_{k}, n_{k}, r\right)}{2 n_{k}}=\frac{1}{2}\left(p \log r-p \log p-2(1-p) \log (1-p)+(r-p) \log \left(1-\frac{p}{r}\right)\right) .
$$

Proof. Recall Stirling's formula [2, p. 52]:

$$
n !=\sqrt{2 \pi n} n^{n} e^{-n} e^{\frac{\theta_{n}}{12 n}} \text { for some } \theta_{n} \in(0,1) \text { and any positive integer } n .
$$

We will use the following version of Stirling's formula

$$
\sqrt{2 \pi n} n^{n} e^{-n}<n !<2 \sqrt{2 \pi n} n^{n} e^{-n} .
$$

Let $\mu_{1}, \ldots, \mu_{r}$ be defined by (4.5). We now estimate from above and below the terms appearing in (4.6) using Stirling's formula.

$$
\begin{gathered}
\frac{m-r}{r}<\mu_{i}<\frac{m+r}{r} \text { for } i=1, \ldots, r \\
\left(\frac{2 \pi(m-r)}{r}\right)^{\frac{r}{2}}\left(\frac{m-r}{r}\right)^{m-r} e^{-m}<\prod_{i=1}^{r} \mu_{i} !<2^{r}\left(\frac{2 \pi(m+r)}{r}\right)^{\frac{r}{2}}\left(\frac{m+r}{r}\right)^{m+r} e^{-m} \\
\left(\frac{2 \pi(r n-m-r)}{r}\right)^{\frac{r}{2}}\left(\frac{r n-m-r}{r}\right)^{r n-m-r} e^{-(r n-m)}< \\
\prod_{i=1}^{r}\left(n-\mu_{i}\right) !<2^{r}\left(\frac{2 \pi(r n-m+r)}{r}\right)^{\frac{r}{2}}\left(\frac{r n-m+r}{r}\right)^{r n-m+r} e^{-(r n-m)}, \\
(2 \pi n)^{\frac{r-2}{2}}(2 \pi(n-m)) n^{(r-2) n}(n-m)^{2(n-m)} e^{-((r-2) n+2(n-m))}< \\
(n !)^{r-2}((n-m) !)^{2}<2^{r}(2 \pi n)^{\frac{r-2}{2}}(2 \pi(n-m)) n^{(r-2) n}(n-m)^{2(n-m)} e^{-((r-2) n+2(n-m))}, \\
1 \leq\left(\begin{array}{c}
m+r-1 \\
r-1
\end{array}\right)<(m+r-1)^{r-1} .
\end{gathered}
$$

We now use these inequalities in (4.6) to estimate the ratio $\frac{1}{2 n_{k}} \log E_{1}\left(m_{k}, n_{k}, r\right)$ where

$$
\lim _{k \rightarrow \infty} m_{k}=\lim _{k \rightarrow \infty} n_{k}=\infty, \lim _{k \rightarrow \infty} \frac{m_{k}}{n_{k}}=p \in[0,1] .
$$


First note that for any polynomial $p(x)$ and any $a \in \mathbb{R} \lim _{k \rightarrow \infty} \frac{\log p\left(m_{k}+a\right)}{n_{k}}=0$. Next observe that $\log (x+a)=\log x+O\left(\frac{1}{x}\right)$ for a fixed $a$ and $x \gg 1$. Let $\frac{m_{k}}{n_{k}}=p_{k}$. Our assumptions yield that $\lim _{k \rightarrow \infty} p_{k}=p$. Then

$$
\begin{aligned}
& \frac{\log \left(n_{k}-\frac{m_{k} \pm r}{r}\right)^{r n_{k}-m_{k} \pm r}}{e^{\left(r n_{k}-m_{k}\right)} n_{k}}=\left(r-p_{k}+O\left(\frac{1}{n_{k}}\right)\right)\left(\log n_{k}+\log \left(1-\frac{p_{k}}{r}\right)+O\left(\frac{1}{n_{k}}\right)\right)-\left(r-p_{k}\right) \\
&=\left(r-p_{k}\right)\left(\log n_{k}+\log \left(1-\frac{p_{k}}{r}\right)\right)-\left(r-p_{k}\right)+o(1), \\
& \frac{\log \left(\frac{m_{k} \pm r}{r}\right)^{m_{k} \pm r}}{e^{m_{k}} n_{k}}=\left(p_{k}+O\left(\frac{1}{n_{k}}\right)\right)\left(\log n_{k}+\log p_{k}-\log r+O\left(\frac{1}{n_{k}}\right)\right)-p_{k} \\
&=p_{k}\left(\log n_{k}+\log p_{k}-\log r\right)-p_{k}+o(1), \\
& \frac{\log n_{k}^{(r-2) n_{k}}\left(n_{k}-m_{k}\right)^{2\left(n_{k}-m_{k}\right)}}{e^{\left((r-2) n_{k}+2\left(n_{k}-m_{k}\right)\right)} n_{k}}=(r-2) \log n_{k}+2\left(1-p_{k}\right)\left(\log n_{k}+\log \left(1-p_{k}\right)\right)-r+2 p_{k} .
\end{aligned}
$$

Subtract the second and the third term from the first one. Note first that the coefficient of $\log n_{k}$ is $\left(r-p_{k}\right)-p_{k}-(r-2)-2\left(1-p_{k}\right)=0$. Hence

$$
\begin{aligned}
\frac{\log E_{1}\left(m_{k}, n_{k}, r\right)}{n_{k}}=( & \left.-p_{k}\right) \log \left(1-\frac{p_{k}}{r}\right)-\left(r-p_{k}\right) \\
& \quad-p_{k} \log p_{k}+p_{k} \log r+p_{k}-2\left(1-p_{k}\right) \log \left(1-p_{k}\right)+r-2 p_{k}+o(1) \\
= & \left(r-p_{k}\right) \log \left(1-\frac{p_{k}}{r}\right)-p_{k} \log p_{k}+p_{k} \log r-2\left(1-p_{k}\right) \log \left(1-p_{k}\right)+o(1) .
\end{aligned}
$$

Finally use the continuity of $\log x$ to deduce $(1.10)$. (Here $0 \log 0=0$.

\subsection{Second measure}

We now deduce (1.10) for a standard probabilistic model on $\mathcal{G}_{\text {mult }}(2 n, r)$ as given in [8]. Let $\mu \in S_{n r}$ be a permutation on $n r$ elements. Let $e_{1}, \ldots, e_{n r}$ be $n r$ edges going from vertices $\{1, \ldots, n\}$ in the group $A$ to vertices $\{1, \ldots, n\}$ to the group $B$. We then assume that $e_{i}$ connects the vertex $\left\lceil\frac{i}{r}\right\rceil$ in group $A$ to $\left\lceil\frac{\mu(i)}{r}\right\rceil$ in group $B$ for $i=1, \ldots, r n$. Note that the vertex $i$ in group $A$ has $r$ edges labeled $r(i-1)+1, \ldots, r i$. It is straightforward to see that each vertex $j$ in the group $B$ has $r$ different edges connected to it, i.e. the equation $j=\left\lceil\frac{\mu(i)}{r}\right]$ has exactly $r$ integer solutions $\mu^{-1}(\{j(r-1)+1, \ldots, j r\})$. Then the probability of such graph is given by $\frac{1}{(r n) !}$. Note if we do not care to label the edges, then an $r$-regular bipartite graph, where each two vertices are connected by at most one edge, is represented by $(r !)^{n}$ such permutations $\mu$. Indeed any vertex $i$ in the first group has $r$ edges labeled $r(i-1)+1, \ldots, r i$ which are connected to it. These edges connect to a set of $r$ vertices $T \subset\{1, \ldots, n\}$. Permuting these $r$ edges out of vertex $i$ between the vertices in the group $T$ has $r$ ! choices, which are all equivalent. Repeat this argument for $i=1, \ldots, n$ to obtain $(r !)^{n}$ choices which gives rise to the same simple graph. Denote by $\nu(n, r)$ the probability measure on $\mathcal{G}(2 n, r)$ induced by these method. 
Lemma 4.5 Let $\nu(n, r)$ be the probability measure defined above. Then

$$
E_{2}(m, n, r):=E_{\nu(n, r)}(\phi(m, G))=\frac{\left(\begin{array}{l}
n \\
m
\end{array}\right)^{2} r^{2 m} m !(r n-m) !}{(r n) !} .
$$

Proof. We adopt the arguments of [10] to our case. First choose subset $\alpha \subset\{1, \ldots, n\}$ of $m$ vertices in the group $A$. There are $\left(\begin{array}{l}n \\ m\end{array}\right)$ choices like that. $\alpha$ induces the set $I=\cup_{i \in \alpha}\{r(i-1)+1, \ldots, i r\}$ of edges of cardinality $r m$. From $I$ choose a set $J$ of $m$ edges, so that $e_{j}, j \in J$ corresponds to the choice of one element in the group $\{r(i-1)+1, \ldots, i r\}$, for each $i \in \alpha$. There are $r^{m}$ of the choices of $J$. Now we want to choose $\mu$ so that $\left\lceil\frac{\mu(j)}{r}\right\rceil, j \in J$ will be a subset of $m$ distinct elements $\beta=\cup_{j \in J}\left\{\beta_{\left\lceil\frac{\mu(j)}{r}\right\rceil}\right\} \subset\{1, \ldots, n\}$. There are $m !\left(\begin{array}{l}n \\ m\end{array}\right)$ such choices of $\beta$. Then $\mu(j) \in\left\{\beta_{\left\lceil\frac{\mu(j)}{r}\right\rceil}(r-1)+1, \ldots, \beta_{\left\lceil\frac{\mu(j)}{r}\right\rceil} r\right\}$ for each $j \in J$. Again there are $r^{m}$ such choices. Thus we chose $\mu$ by determining the image of the elements in $J$ in $\{1, \ldots, n r\}$, which is denoted by $\mu(J)$. The rest of the elements $\{1, \ldots, r n\} \backslash J$ are mapped to $\{1, \ldots, r n\} \backslash \mu(J)$. The number of choices here is $(n r-m)$ !. Multiply all these choices to get the numerator of the right-hand side of (4.10). Divide this number of choices by the number of permutations of $\{1, \ldots, r n\}$ to deduce the lemma.

Using the methods in the proof of Theorem 4.4 we get the

\section{Corollary 4.6}

$$
\begin{aligned}
\lim _{k \rightarrow \infty} \frac{\log E_{2}\left(m_{k}, n_{k}, r\right)}{2 n_{k}}= & \frac{1}{2}\left(p \log r-p \log p-2(1-p) \log (1-p)+(r-p) \log \left(1-\frac{p}{r}\right)\right), \\
& \text { if } \lim _{k \rightarrow \infty} n_{k}=\lim _{k \rightarrow \infty} m_{k}=\infty, \quad \text { and } \quad \lim _{k \rightarrow \infty} \frac{m_{k}}{n_{k}}=p \in[0,1] .
\end{aligned}
$$

\section{$5 \quad$ Asymptotic Lower Matching Conjecture}

For integers $2 \leq r, 1 \leq m \leq n$ let $\mu(m, n, r)$ be defined by $(1.5)$. Fix $p \in(0,1]$ and consider two increasing sequences $\left\{m_{k}\right\},\left\{n_{k}\right\}$ as in Theorem 4.4. Let $\operatorname{low}_{r}(p)$ be the largest real number (possibly $\infty$ ) for which one always has the inequality

$$
\liminf _{k \rightarrow \infty} \frac{\log \mu\left(m_{k}, n_{k}, r\right)}{n_{k}} \geq \operatorname{low}_{r}(p), \quad p \in(0,1] .
$$

So $\operatorname{low}_{r}(p)$ is the limit infimum over all possible values given by the left-hand side of (5.1). Hence $g h_{r}(p) \geq \operatorname{low}_{r}(p)$ for all $p \in[0,1]$.

The equality (1.11) and (1.7) imply the equality

$$
\operatorname{low}_{r}(1)=\log \frac{(r-1)^{r-1}}{r^{r-2}} .
$$

(See for details $[3, \S 5]$ and $[4, \S 3]$.) Hence, in the first version of this paper in 2005 we conjectured the Asymptotic Lower Matching Conjecture, abbreviated here as ALMC. 
Conjecture 5.1 (ALMC) For any $2 \leq r \in \mathbb{N}, p \in(0,1) \operatorname{low}_{r}(p)$ is equal to the righthand side of (1.10):

$$
\operatorname{low}_{r}(p)=p \log r-p \log p-2(1-p) \log (1-p)+(r-p) \log \left(1-\frac{p}{r}\right)
$$

Theorem $5.2 \operatorname{low}_{2}(p)=g h_{2}(p)$ for all $p \in[0,1]$, where $g h_{2}(p)$ is defined by (1.12).

Proof. Theorem 2.2 yields that

$$
\mu(m, n, 2)=\phi\left(m, C_{2 n}\right)=\left(\begin{array}{c}
2 n-m \\
m
\end{array}\right)+\left(\begin{array}{c}
2 n-m-1 \\
m-1
\end{array}\right) .
$$

Use Stirling's formula as in the proof of Theorem 4.4 to deduce the equality $\operatorname{low}_{2}(p)=$ $g h_{2}(p)$.

Friedland and Gurvits $[3, \S 5]$ have proved the following theorem

Theorem 5.3 Let $r \geq 3, s \geq 1$ be integers. Let $B_{n}, n=1,2, \ldots$ be a sequence of $n \times n$ doubly stochastic matrices, where each column of each $B_{n}$ has at most $r$-nonzero entries. Let $k_{n} \in[0, n], n=1,2, \ldots$ be a sequence of integers with $\lim _{n \rightarrow \infty} \frac{k_{n}}{n}=p \in(0,1]$. Then

$$
\begin{aligned}
\liminf _{n \rightarrow \infty} \frac{\log \operatorname{perm}_{k_{n}} B_{n}}{2 n} \geq & \frac{1}{2}(-p \log p-2(1-p) \log (1-p))+ \\
& \frac{1}{2}\left((r+s-1) \log \left(1-\frac{1}{r+s}\right)-(s-1+p) \log \left(1-\frac{1-p}{s}\right)\right) .
\end{aligned}
$$

Also, the Asymptotic Lower Matching Conjecture 5.1 holds for $p_{s}=\frac{r}{r+s}, s=0,1,2, \ldots$.

Small lower bounds for $\operatorname{low}_{r}(p)-g h_{r}(p)$ for all values of $p \in[0,1]$ are given in $[4, \S 3]$. Use Stirling's formula, as in the proof of Theorem 4.4 to deduce:

Proposition 5.4 Assume that the inequality (1.8) holds for all $m \in[2, n] \cap \mathbb{N}, 3 \leq$ $r \in \mathbb{N}$ and all $n \geq N(r)$. Then ALMC holds.

\section{Maximal matchings in $\mathcal{G}_{\text {mult }}(2 n, r)$ and $\mathcal{G}(2 n, r)$}

Proposition 6.1 Let $G=\left(V_{1} \cup V_{2}, E\right)$ be a bipartite multigraph where $V_{1}, V_{2}$ are the two groups of the set of vertices. Let $\# V_{1}=n$ and assume that the degree of each vertex in $V_{1}$ is $r \geq 2$. Then

$$
\phi(m, G) \leq\left(\begin{array}{c}
n \\
m
\end{array}\right) r^{m} \text { for each } m=1, \ldots, n .
$$

Assume that $\# V_{2}=n$. Then for $m \geq 2$ equality holds if and only if $G=n H_{r}$, i.e. $A(G)=r I_{n}$. In particular (1.6) holds. 
Proof. Let $M \subset E$ be an $m$-matching. Then $M$ covers exactly $U \subset V_{1}$ vertices of cardinality $m$. Then number of choices of $U$ is $\left(\begin{array}{c}n \\ m\end{array}\right)$. Let $v \in U$. Then $v$ can be covered by $r$ edges. Hence (6.1) holds.

Suppose that $m \geq 2$ and $\# V_{2}=n$. Let $w \in V_{2}$ and assume that $w$ is connected to two distinct vertices $v_{1}, v_{2} \in V_{1}$ by the edges $e_{1}, e_{2}$. Then these two edges cannot appear together in any $m$-matchings. Hence for this $G$ one has a strict inequality in (6.1). Thus, if $\# V_{2}=n$ and $m \geq 2$ equality holds in (6.1) if and only if $G=n H_{r}$.

The inequality (6.1) for $G \in \mathcal{G}(2 n, r)$ was used in [4]. In the first version of this paper we conjectured that $\Lambda(m, n, r):=\max _{G \in \mathcal{G}(2 n, r)} \phi(m, G)$ is achieved for the maximal graph $q K_{r, r}$, i.e. disjoint unions of $q$ complete bipartite graphs on $2 r$ vertices, if $n \equiv 0 \bmod 4$.

We state a generalization of the conjecture (1.4) for $\mathcal{G}(2 n, r)$ when $n$ is not divisible by $r$ :

$$
\phi(m, G) \leq \phi\left(m,\left\lfloor\frac{n}{r}\right\rfloor K_{r, r} \cup\left(n-r\left\lfloor\frac{n}{r}\right\rfloor\right) H_{r}\right) \text { for any } G \in \mathcal{G}(2 n, r) .
$$

Theorem 2.2 yields that the validity of the conjecture (6.2) for $r=2$. See [4] for the asymptotic version of the conjectured inequality (6.2).

\section{Computational results}

\subsection{The Lower Matching Conjecture for finite graphs}

For small $r$-regular bipartite graphs on $2 n$ vertices we have tested the following finite analogue of the lower matching conjecture.

$$
\phi(G, m) \geq \varphi(n, r, m)=\left(1+\frac{1}{r n}\right)^{r n-1}\left(1-\frac{m}{r n}\right)^{r n-m}\left(\frac{m r}{n}\right)^{m}\left(\begin{array}{c}
n \\
m
\end{array}\right)^{2}
$$

Note that as $n$ grows this bound is asymptotically exact for 1-edge matchings, and the convergence is faster for larger $r$.

In order to test the conjecture we computed the matching generating polynomials for all bipartite regular graphs on $2 n \leq 20$ vertices and compared with the bound. The bound held for all such graphs.

For $2 n \geq 21$ the number of bipartite regular graphs is too large for a complete test of all graphs, the computing time for each graph also grows exponentially, so we instead tested the conjecture for graphs of higher girth. The combinations of degree and girth are given in Figure 7.1. Again the conjecture held for all such graphs.

\subsection{The Upper Matching Conjecture for Cubic graphs}

We have checked the upper matching conjecture for $r=3$ and $2 n$ up to 24 by computing the matching generating polynomials for all connected bipartite cubic graphs, up to isomorphism, in this range. For $2 n=6$ and $2 n=8$ there is only one cubic bipartite graph of the given size: $K_{3,3}$ and the 3 -dimensional hypercube $Q_{3}$ respectively. For $2 n=10$ there 


\begin{tabular}{|r|c|c|c|c|c|c|c|c|}
\hline $2 n$ & 22 & 24 & 26 & 28 & 30 & 32 & 34 & 36 \\
\hline$r=3$ & 6 & 6 & 6 & 6 & 6 & 8 & 8 & 8 \\
$r=4$ & & & 6 & 6 & 6 & 6 & 6 & \\
\hline
\end{tabular}

Figure 1: Lower bound for the girth of the regular bipartite graphs of order greater than 20 used in our tests. An empty entry means that no graphs of that order and degree were used.

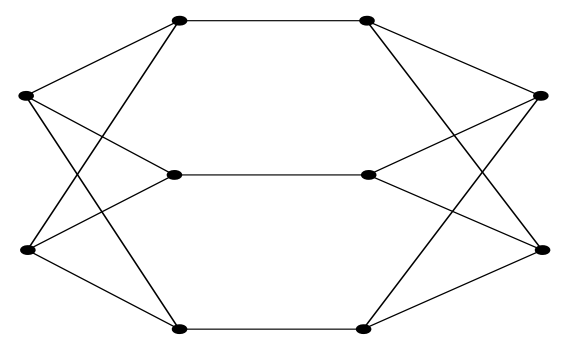

Figure 2: $G_{1}$

are two graphs to consider and they turn out to have incomparable matching generating functions. The first graph $G_{1}$ is shown in Figure 2 and the second graph is the 10 vertex Möbius ladder $M_{10}$. ( $M_{10}$ consists of two copies of path of length 5: $1-2-3-4-5$, denoted by $\left(P_{5}, 1\right)$ and $\left(P_{5}, 2\right)$, where first one connects $(i, 1)$ and $(i, 2)$ by an edge for $i=1, \ldots, 5$, and then one connects $(1,1)$ with $(5,2)$ and $(1,2)$ with $(5,1)$.)

Their matching generating polynomials are:

$$
\begin{aligned}
\psi\left(x, G_{1}\right) & :=1+15 x+75 x^{2}+145 x^{3}+96 x^{4}+12 x^{5} \\
\psi\left(x, M_{10}\right) & :=1+15 x+75 x^{2}+145 x^{3}+95 x^{4}+13 x^{5} .
\end{aligned}
$$

For $2 n$ from 12 to 24 the extremal graphs, with the maximal $\phi(l, G)$, are for the form

$$
\begin{array}{ll}
\frac{2 n}{6} K_{3,3} & \text { if } 6 \mid 2 n \\
\frac{2 n-8}{6} K_{3,3} \cup Q_{3} & \text { if } 6 \mid(2 n-2) \\
\frac{2 n-10}{6} K_{3,3} \cup\left(G_{1} \text { or } M_{10}\right) & \text { if } 6 \mid(2 n-4)
\end{array}
$$

So for $2 n=10,22$ we do not have a unique extremal graph, which maximizes all $\phi(l, G)$. It seems natural to conjecture that the three graph families given here together make up all the extremal graphs for all $n$.

\section{Exact values for small matchings}

In this section we derive exact expressions for $\phi(G, m)$ for $m \leq 4$ and compare the results with our conjectured bounds. After this paper was accepted for publication Ian Wanless 
contacted us to point out that in fact these expressions were derived in [12] for $m \leq 5$. The upcoming paper [14] further explores an algorithmic method for computing expressions of this type.

Theorem 8.1 Assume that $G$ is a bipartite $r$-regular graph on $2 n$ vertices and that $G$ contains $a_{4}(G)$ 4-cycles, then

1. $\phi(G, 1)=r n$

2. $\phi(G, 2)=\left(\begin{array}{c}n r \\ 2\end{array}\right)-2 n\left(\begin{array}{l}r \\ 2\end{array}\right)=\frac{r n(r n-(2 r-1))}{2}$

3. $\phi(G, 3)=\left(\begin{array}{c}n r \\ 3\end{array}\right)-2 n\left(\begin{array}{l}r \\ 3\end{array}\right)-n r(r-1)^{2}-2 n\left(\begin{array}{l}r \\ 2\end{array}\right)(n r-2 r-(r-2))$

4. $\phi(G, 4)=p_{1}(n, r)+a_{4}(G)$ where

$$
p_{1}(n, r)=\frac{n^{4} r^{4}}{24}+\frac{n^{3} r^{3}}{4}(1-2 r)+\frac{n^{2} r^{2}}{24}\left(19-60 r+52 r^{2}\right)+n r\left(\frac{5}{4}-5 r+7 r^{2}-\frac{7 r^{3}}{2}\right) .
$$

\section{Proof.}

1. This is just the number of edges in $G$.

2. There are $\left(\begin{array}{c}n r \\ 2\end{array}\right)$ 2-edge subsets of $E(G)$. Such a subset is not a matching if it forms a three vertex path $P_{3}$. Given a $P_{3} \subset G$ we call the vertex of degree 2 the root. The number of $P_{3}$ 's in $G$ is $2 n\left(\begin{array}{l}r \\ 2\end{array}\right)$, since there are $2 n$ choices for the root vertex and at that vertex there are $\left(\begin{array}{l}r \\ 2\end{array}\right)$ ways of choosing two edges.

3. As in the previous case three edges in $G$ can be chosen in $\left(\begin{array}{c}n r \\ 3\end{array}\right)$ ways. There are three three-edge subgraphs which are not a matching, depicted in Figure 3. The number of 4-vertex stars, $2 n\left(\begin{array}{l}r \\ 3\end{array}\right)$, is counted as in the previous case. The number of $P_{4}$ 's is $n r(r-1)^{2}$, since the middle edge can be chosen in $n r$ ways and the two remaining edges in $r-1$ ways each. The number of subgraphs $P_{3} \cup K_{2}$ is $2 n\left(\begin{array}{l}r \\ 2\end{array}\right)(n r-2 r-(r-2))$, since the $P_{3}$ can be chosen as in the previous case, and the $K_{2}$ can be chosen among the $(n r-2 r-(r-2))$ edges which are not incident with any of the vertices in the $P_{3}$.

4. Let $\mathcal{E}_{4}(G)$ be the subset of all subgraphs of $G \in \mathcal{G}(2 n, r)$ consisting of 4 edges. Then $\# \mathcal{E}_{4}(G)=\left(\begin{array}{c}n r \\ 4\end{array}\right)$. For $H \in \mathcal{E}_{4}(G)$ let $l(H) \geq 0$ be the number of $P_{3}$ subgraphs of $H$. $H \in \mathcal{E}_{4}(G)$ is a matching if and only $l(H)=0$. There are $2 n\left(\begin{array}{c}r \\ 2\end{array}\right)\left(\begin{array}{c}n r-2 \\ 2\end{array}\right)$ graphs $H \in \mathcal{E}_{4}(G)$ which contain at least one $P_{3}$ with a specified root vertex, since there are $2 n$ ways to place the root of a $P_{3}$ and $\left(\begin{array}{c}n r-2 \\ 2\end{array}\right)$ ways to choose the remaining two edges. Note that $2 n\left(\begin{array}{c}r \\ 2\end{array}\right)\left(\begin{array}{c}n r-2 \\ 2\end{array}\right)=\sum_{H \in \mathcal{E}_{4}(G), l(H) \geq 1} l(H)$. Thus, the correct number of 4 -matches is

$$
\left(\begin{array}{c}
n r \\
4
\end{array}\right)-2 n\left(\begin{array}{l}
r \\
2
\end{array}\right)\left(\begin{array}{c}
n r-2 \\
2
\end{array}\right)+\sum_{H \in \mathcal{E}_{4}, l(H)>1}(l(H)-1)
$$



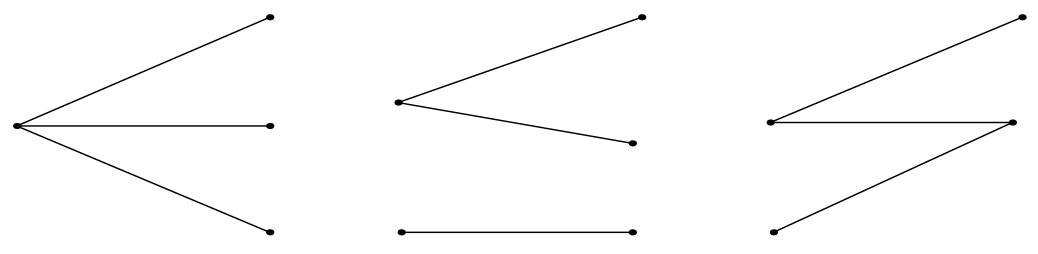

Figure 3: The 3 edge subgraphs

In Figure 4 we display all subgraphs $H$ with $l(H)>1$. The number of copies of each graph and its number of $P_{3}$ 's is

S1 Number: $2 n\left(\begin{array}{l}r \\ 4\end{array}\right), P_{3}$ 's $\left(\begin{array}{l}4 \\ 2\end{array}\right)$

S2 Number: $2 n\left(\begin{array}{l}r \\ 3\end{array}\right) 3(r-1), P_{3}$ 's $1+\left(\begin{array}{l}3 \\ 2\end{array}\right)$

S3 Number: $\left.2 n\left(\begin{array}{l}r \\ 3\end{array}\right)(n r-4 r+3)\right), P_{3}$ 's $\left(\begin{array}{l}3 \\ 2\end{array}\right)$

S4 Number: $2 n\left(\begin{array}{c}r \\ 2\end{array}\right)(r-1)^{2}-4 a_{4}(G), P_{3}$ 's 3

S5 Number: $a_{4}(G), P_{3}$ 's 4

S6 Number: $n(n-2)\left(\begin{array}{l}r \\ 2\end{array}\right)^{2}-\frac{1}{2}(\# S 2), P_{3}$ 's 2

S7 Number: $2\left(\begin{array}{c}n \\ 2\end{array}\right)\left(\begin{array}{l}r \\ 2\end{array}\right)^{2}-2 a_{4}(G)-(\# S 4), P_{3}$ 's 2

S8 Number: $\left(n r(r-1)^{2}-4 a_{4}(G)\right)(n r-4 r+3)+4 a_{4}(G)(n r-4 r+4), P_{3}$ 's 2

Use the above formulas in (8.2) to obtain a rather messy expression for $\phi(G, 4)$. After some simplification we obtain the formula we have in the theorem. that

If we compute the limits of $\frac{\phi(G, m)}{\varphi(n, r, m)}$ for the values of $m$ used in Theorem 8.1 we find

$$
\begin{gathered}
\lim _{n \rightarrow \infty} \frac{\phi(G, 1)}{\varphi(n, r, 1)}=1 \\
\lim _{n \rightarrow \infty} \frac{\phi(G, 2)}{\varphi(n, r, 2)}=\frac{e}{2}=1.359 \\
\lim _{n \rightarrow \infty} \frac{\phi(G, 3)}{\varphi(n, r, 3)}=\frac{2 e^{2}}{9}=1.642 \ldots \\
\lim _{n \rightarrow \infty} \frac{\phi(G, 4)}{\varphi(n, r, 4)}=\frac{3 e^{3}}{32}=1.883 \ldots
\end{gathered}
$$

This indicates that there exists some stronger form of the lower bound for finite graphs, but if the ALMC is true this additional factor will be subexponential in $n$, possibly just a function of $m$.

In the expression for $\phi(G, 4)$ the number of 4-cycles appeared as the first structure in the graph, apart from $n$ and $r$, which affects the number of matchings. The maximum possible value of $a_{4}(G)$ can be found. 

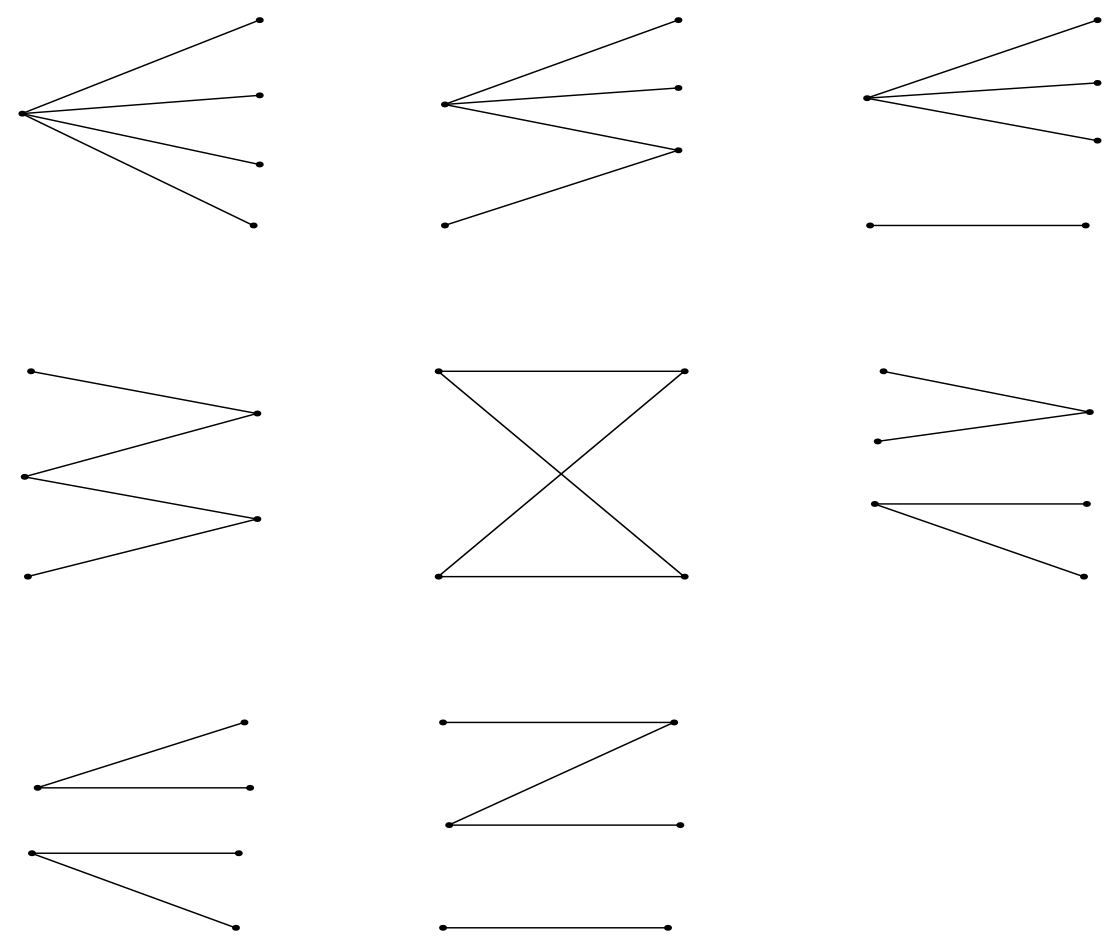

Figure 4: The 4 edge subgraphs

Lemma 8.2 Let $G$ be an $r$-regular bipartite graph on $2 n$ vertices, with $r \geq 2$. Then

$$
a_{4}(G) \leq \frac{n r(r-1)^{2}}{4}
$$

Equality holds if and only if $n=q r$ and $G$ is the disjoint union of $q K_{r, r}$.

In [13] a conjecture regarding the structure of graphs with maximal values of $a_{4}(G)$ for general $n$ is given.

Proof. Given an edge $e$ in $G$, the largest number of 4-cycles which can contain $e$ as the central edge is $(r-1)^{2}$. Indeed, the number of $P_{4}$ 's which contain $e$ is $(r-1)^{2}$. Each $P_{4}$ can be completed to a 4 cycle if and only if $e$ is an edge in a connected component of $G$ equal to $K_{r, r}$. Since $G$ has $n r$ edges and each 4 cycle consists of 4 edges we deduce the inequality (8.3). Assume equality in (8.3). Then every edge belongs to a $K_{r, r}$ component of $G$. Hence $G=q K_{r, r}$.

This has some simple but nice corollaries.

Corollary 8.3 The upper and lower matching conjectures are true for $m \leq 4$.

In [15] the distribution of the number of short cycles in a bipartite random regular graph was determined, and applying that result here we find that, 
Corollary 8.4 For random graphs from $\mathcal{G}(2 n, r)$ we have that $\phi(4, G)-p_{1}(n, r)$ converges in distribution to a Poisson random variable with expectation $\frac{(r-1)^{4}}{4}$.

This means that the expected number of 4-edge matchings in a random graph is only a fixed constant larger than the minimum possible, and also only a fixed constant larger than the lower matching conjecture.

Acknowledgement: We would like to thank the referee for useful remarks and Ian Wanless for pointing out several important references. This research was conducted using the resources of Centre for Parallel Computers (PDC).

\section{References}

[1] L.M. Bregman, Some properties of nonnegative matrices and their permanents, Soviet Math. Dokl. 14 (1973), 945-949.

[2] W. Feller, An Introduction to Probability Theory and Its Applications, vol. I, J. Wiley \& Sons, 1958.

[3] S. Friedland and L. Gurvits, Lower bounds for partial matchings in regular bipartite graphs and applications to the monomer-dimer entropy, Combinatorics, Probability and Computing, 17 (2008). (Previous version: "Generalized Friedland-Tverberg inequality: applications and extensions", arXiv:math/0603410 v2, 24 Aug 2006.)

[4] S. Friedland, E. Krop, P.H. Lundow and K. Markström, Validations of the Asymptotic Matching Conjectures, arXiv:math/0603001 v2, 21 Jun 2007, To appear in Journal of Statistical Physics.

[5] S. Friedland and U.N. Peled, Theory of Computation of Multidimensional Entropy with an Application to the Monomer-Dimer Problem, Advances of Applied Math. 34(2005), 486-522.

[6] L. Gurvits, Hyperbolic polynomials approach to van der Waerden/Schrijver-Valiant like conjectures: Sharper bounds, simpler proofs and algorithmic applications, STOC'06: Proceedings of the 38th Annual ACM Symposium on Theory of Computing, 417-426, ACM, New York, 2006. arXIv:math.CO/0510452 v3, 12 Jan 2006.

[7] S. Janson, T. Łuczak and A. Ruciński, Random Graphs, John Wiley, 2000.

[8] L. Lovász and M.D. Plummer, Matching Theory, North-Holland Mathematical Studies, vol. 121, North-Holland, Amsterdam, 1986.

[9] B. D. McKay and I. M. Wanless, Maximising the permanent of $(0,1)$ matrices and the number of extensions of Latin rectangles, Electron. J. Combin. 5 (1998), R11.

[10] A. Schrijver, Counting 1-factors in regular bipartite graphs, J. Comb. Theory B 72 (1998), 122-135.

[11] I. M. Wanless, Addendum to Schrijver's work on minimum permanents, Combinatorica 26 (2006), 743-745. 
[12] I. M. Wanless, The Holens-Dokovic conjecture on permanents fails, Linear Algebra Appl 286 (1999), 273-285.

[13] I. M. Wanless, Maximising the permanent and complementary permanent of $(0,1)$ matrices with constant line sum, Discrete Math. 205 (1999), 191-205.

[14] I. M. Wanless, Counting matchings and tree-like walks in regular graphs, manuscript 2007.

[15] N.C. Wormald, Some Problems in the Enumeration of Labelled Graphs, PhD thesis, University of Newcastle, 1978. 